\title{
Novel dynamical symmetries of asymmetrically doubly excited two-electron atoms
}

\author{
K Richter $\dagger$, J S Briggs $\dagger$, D Wintgen $\dagger$ and E A Solov'ev $\ddagger$ \\ $\dagger$ Fakultät für Physik, Hermann-Herder-Strasse 3, 7800 Freiburg, Federal Republic of \\ Germany \\ $\ddagger$ Department of Theoretical Physics, St Petersburg University, St Petersburg, Russia
}

Received 3 February 1992, in final form 16 June 1992

\begin{abstract}
We study a new class of long-lived resonances of doubly excited two-electron atoms. The states are characterized by a highly polarized inner electron located near the axis between the nucleus and a dynamically localized outer electron. Classical mechanics studies prove the stability of the corresponding classical motion and allow for EBK quantization to obtain semiclassical energies. The resonance states are treated further within the framework of a single-channel adiabatic approximation, where the inner electron is prescribed by a polarized molecular type wavefunction. The adiabatic energies as well as the semiclassical results are in good agreement with resonance energies obtained by highly accurate solutions of the full three-body Schrödinger equation. There is a one-to-one correspondence between approximate quantum numbers derived from the semiclassical and from the adiabatic approach, both of which explain the nodal structures of the $a b$ initio quantum wavefunctions reflecting the approximate dynamical symmetries of the problem. Decay mechanisms and the resulting widths of the resonances are discussed. The dependence of these configurations on the nuclear charge $Z$ is examined.
\end{abstract}

\section{Introduction}

The non-separability of the three-body Coulomb problem becomes evident in the case of highly doubly excited atoms or ions, where the electron-electron interaction is of comparable importance to the electron-ion interaction. The effect of inter-electron repulsion, i.e. electron correlation, typically leads to the breakdown of independent particle approaches and has focused interest on the search for approximate symmetries using collective coordinates of the three particles.

Experimentally, much progress has been reported recently in the spectroscopy of highly doubly excited atoms. Some experiments use single-photon excitation from the ground state to study pure three-body Coulomb systems such as $\mathrm{H}^{-}$(Harris et al 1990) and He (Domcke et al 1991), while other experiments involve multistep laser excitations to populate doubly excited states in alkaline earth atoms (Camus et al 1989, Roussel et al 1990, Jones and Gallagher 1990, Eichmann et al 1990, 1992). The development of sophisticated laser excitation schemes of alkaline earth atoms allows one to prepare non-core-penetrating doubly excited states and to examine helium-like three-body systems spectroscopically with the high resolution obtainable with lasers (Eichmann et al 1992).

Due to the intrinsic non-separability of the problem, there exists no symmetry classification in terms of globally valid quantum numbers which would then allow an 
overall description of the huge variety of doubly excited states occurring. Theoretically, progress has been made in uncovering approximate symmetries for particular classes of states. The intra-shell resonances (composed of two excited electrons with roughly equally shared energy and radial expectation values $\left.\left\langle r_{1}\right\rangle \approx\left\langle r_{2}\right\rangle\right)$ were classified by introducing new sets of approximate quantum numbers based on collective particle motion (Herrick and Kellman 1980, Herrick 1983, Lin 1986, Feagin and Briggs 1986, 1988, Rost and Briggs 1991). For extremely asymmetric configurations $\left\langle r_{1}\right\rangle \gg\left\langle r_{2}\right\rangle$, asymptotic expansions such as the dipole expansion of the inter-electron potential (Gailitis and Damburg 1963, Herrick 1983, Rost and Briggs 1991) or the 'frozen planet approximation' (Eichmann et al 1990) have been applied.

In two recent publications (Richter and Wintgen 1990a, 1991) the existence of a novel class of strongly correlated electron states ('planetary atom' states (Percival 1977)) which show moderate asymmetric electron excitation was predicted and verified. These resonance states are described in detail in the present paper and the internal symmetries of these states are uncovered. They are composed of a strongly polarized (inner) electron localized along the axis connecting the nucleus and the outer electron which is dynamically localized near some fixed radial distance. The slow motion of the outer electron can be interpreted as a trapping mechanism in the flat minimum of an effective potential. The localization of the outer electron results from the combined attractive nuclear potential and the repulsive force induced by the charge density of the polarized inner electron. The resonance states have the following pronounced properties:

(i) distinct angular and radial correlations;

(ii) extremely small (particle-) decay widths;

(iii) quasi-separability of the wavcfunctions in collective semiclassical and molecular coordinates.

In this paper we extend the (semi-) classical and quantum mechanical calculations of the previous letter (Richter and Wintgen 1991). In addition, we present an adiabatic quantum mechanical treatment of the resonances. Guided by the classical analogue, we treat the distance $r_{1}$ between the nucleus and the localized outer electron as an adiabatic parameter and solve the Schrödinger equation for the inner electron exposed to the combined Coulomb potentials of the two fixed charges $\mathrm{He}^{2+}$ and $\mathrm{e}^{-}$. For sufficiently high excitation of the inner electron the resulting Born-Oppenheimer potentials exhibit a secondary minimum in which slow vibrational motion of the outer electron takes place. This molecular orbital approach gives new approximate quantum numbers, which are related to the separability of the two-centre problem for the inner electron.

The strong radial and angular correlations of the electrons lead to the mixing of all single-particle angular momenta $l_{i}$ resulting in the formation of a molecular- (or Stark-) type inner electronic wavefunction and a vibrational, highly non-hydrogenic wavefunction for the outer electron. The resonances are associated with classical phase space regions, where the three-body Coulomb system becomes nearly integrable, which reflects the, underlying dynamical symmetry for this particular class of resonances.

The resonances exist for arbitrary finite nuclear charges $Z>1$. Their existence is inherently tied to the repulsive electron-electron interaction. In particular, they do not possess a limiting independent-particle motion. Hence perturbation theory in $1 / Z$ starting from the independent-particle limit should fail in describing these states.

The paper is organized as follows. Section 2 describes the relevant classical mo- 
tion and the semiclassical predictions for the energies and for the nodal structures of associated wavefunctions. In section 3 we develop an appropriate quantum adiabatic treatment and calculate potential curves for a single-channel adiabatic approximation. Section 4 describes the ab initio quantum mechanical procedure to solve the full three-body Coulomb problem using complex scaling techniques. We compare our results for resonance energies and expectation values obtained by the different methods in section 5. In addition, we discuss the nodal structure of the $a b$ initio quantum wavefunctions, which uncovers their approximate separability in molecular coordinates as well as in the local coordinates of the fundamental classical periodic orbit. In section 6 we discuss possible (particle-) decay mechanisms and report on our calculated decay widths. Section 7 discusses the dependence of our results (presented in sections $2-6$ for helium) for other nuclear charges $Z$. Finally, we comment on related work in section 8 . The results are summarized and concluded in section 9.

\section{Classical mechanics and semiclassical quantization}

We first give a (semi-) classical analysis of the relevant electron pair motion. This probably gives the most insight into the underlying dynamical properties, which will be treated fully quantum mechanically in the subsequent chapters.

The non-relativistic Hamiltonian of a two-electron atom (or ion) with charge $Z$ is given by (atomic units used throughout the paper)

$$
H=\frac{p_{1}^{2}+p_{2}^{2}}{2}+\frac{1}{2 M}\left(p_{1}+p_{2}\right)^{2}-\frac{Z}{r_{1}}-\frac{Z}{r_{2}}+\frac{1}{r_{12}} .
$$

$r_{1}$ and $r_{2}$ are the electron distances from the nucleus, and $r_{12}$ is the inter-electron distance. The second term including the non-trivial mass polarization term proportional to $p_{1} \cdot p_{2}$ vanishes in the limit of infinite nuclear mass $M$. We consider the nucleus as having infinite mass for the dynamics, while the effects of finite mass are taken into account via first-order perturbation theory. All the conclusions drawn in the present paper hold for finite mass typical of nuclei, and the perturbative inclusion of the mass polarization term is consistent with the accuracy reported.

We will mainly focus on states with total angular momentum $\boldsymbol{L}=0$. Then the motion is confined to a fixed plane in configuration space and the Hamiltonian reduces essentially to three (coupled) degrees of freedom. It is convenient to exploit a scaling property of the Hamiltonian (1). Expressed in energy scaled distances $r^{\text {sc }}$, momenta $p^{\text {sc }}$, and time $T^{\text {sc }}$,

$$
\begin{aligned}
& r^{\mathrm{sc}}=(-E) r \\
& p^{\mathrm{sc}}=(-E)^{-1 / 2} p \\
& T^{\mathrm{sc}}=(-E)^{3 / 2} T
\end{aligned}
$$

the Hamiltonian becomes energy independent and we can fix $E$ at the value -1 in equation (1). Details of our numerical procedure to solve the classical equations of motion associated with the Hamiltonian (1) (in particular the regularization of particle collisions) are given in Richter and Wintgen (1990b) and Richter (1991).

Consider a collinear arrangement of a nucleus $Z$ and of two electrons, both being on the same side of the nucleus. The fundamental periodic motion of such 
a configuration is a coherent oscillation of both electrons with the same frequency but, as it turns out, with large differences in their individual radial amplitudes and velocities as depicted in figure $1(a)$ for helium $(Z=2)$ : The outer electron appears to stay nearly frozen at some fixed radial distance. For this reason we label the orbits as frozen-planet configurations. We emphasize that the localization of the outer electron is a dynamical effect and does not rely on the validity of the so-called frozen-planet approximation (Eichmann et al 1990) in which the outer electron is (artificially) fixed.

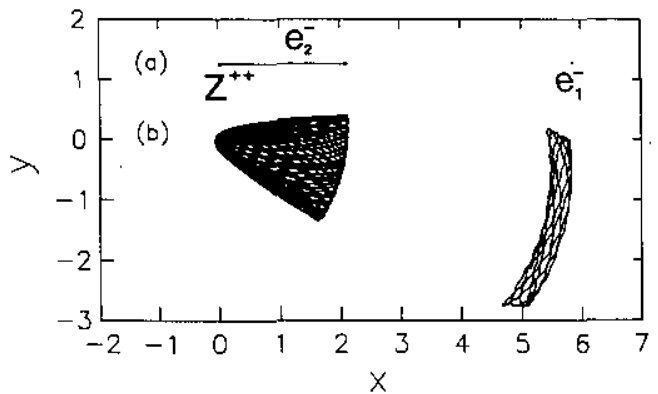

Figure 1. The radial extents of the electrons for $(a)$ the periodic trajectory and $(b)$ a non-periodic but regular trajectory in its neighbourhood.

The significance of a periodic orbit for the corresponding quantized system depends essentially on the structure of the classical phase space in the vicinity of the orbit (Gutzwiller 1990). The periodic trajectory of figure $1(a)$ is linearly stable with respect to variations in the initial conditions. This is demonstrated in figure $1(b)$, which shows the resulting regular motion of the electrons when they are initially in a slightly off-collinear arrangement. The inner electron then moves on perturbed Kepler ellipses around the nucleus, while the outer electron remains trapped at large radial distances following the slow angular precession of the inner electron. The localization of the outer electron rests upon the inter-electron repulsion for small distances of the electrons and upon the asymptotical dominance of the nuclear attraction.

For any collinear configuration the electron pair motion is confined to the threedimensional energy shell of a four-dimensional subspace of the full phase space. It is then convenient to visualize the phase space structure by taking Poincare surfaces of section. Such a section is shown for helium in figure 2. The phase space position $\left\{r_{1}, p_{1}\right\}$ of the outer electron is monitored each time the inner electron approaches the nucleus $\left(r_{2}=0\right)$. The periodic orbit shown in figure $1(a)$ appears as the elliptic fixed point in the centre of the extended torus structure. The non-closed manifolds surrounding the tori represent (regular) trajectories for which the outer electron ionizes. Recalling the additional stability of the bending degree of freedom (i.e. motion off the collinear arrangement), the fundamental periodic orbit of figure 1 is embedded in a fully six-dimensional island of stability in phase space.

The near-integrability of the three-body Coulomb problem for asymmetric configurations as shown in figure 1 is a remarkable property, which nevertheless was only recently uncovered (Richter and Wintgen 1990a, 1991, Richter et al 1991). It is also unexpected and surprising that these classical configurations are extremely stable against autoionization, which is allowed energetically. In contrast, 'symmetric' collinear configurations with the electrons localized on opposite sides of the nucleus 


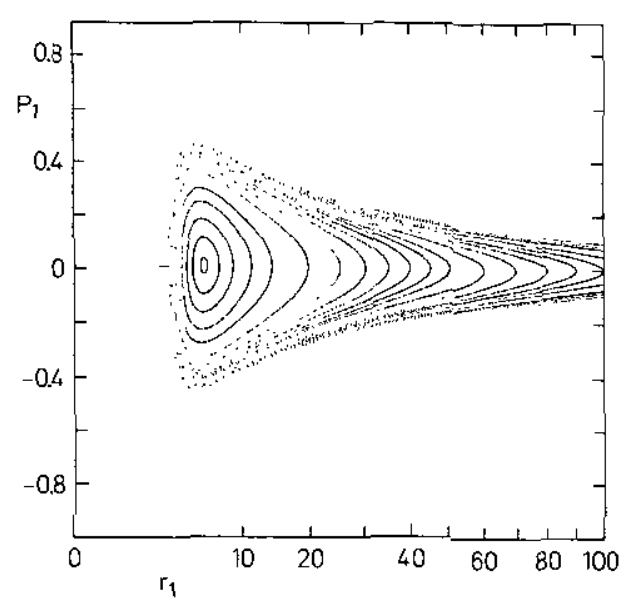

Figure 2. Poincaré surface of section for collinear configurations of the electrons (both electrons on the same side of the nucleus). The radius $r_{1}$ and the radial momentum $p_{1}$ of the outer electron is monitored whenever the inner electron approaches the nucleus $\left(r_{2}=0\right)$.

turn out to be fully chaotic (Ezra et al 1991, Blümel and Reinhardt 1991) and quickly ionize.

Approximate EBK quantization of the tori surrounding the periodic orbit gives (Miller 1975)

$$
S=\frac{S^{\mathrm{sc}}}{\sqrt{-E}}=n+\frac{1}{2}+2\left(k+\frac{1}{2}\right) \gamma_{1}+\left(l+\frac{1}{2}\right) \gamma_{2} .
$$

$S^{\text {sc }}=1.49150$ is the (scaled) action of the periodic orbit for helium $(Z=2)$. The winding numbers $\gamma_{1}=0.46164$ and $\gamma_{2}=0.06765$ describe the behaviour of nearby trajectories. Quantitatively, they represent the contribution of the motion transverse to the periodic orbit (Gutzwiller 1990) to the accumulated action along an arbitrary classical path. The winding numbers enter the eigenvalues of the stability matrix of the periodic orbit after one period (Eckhardt and Wintgen 1991). The factor of 2 appearing in connection with $\gamma_{1}$ takes into account the boundary condition for the Green function in the particular symmetry plane of collinear configurations (Wintgen et al 1992). The semiclassical quantum numbers $n, k$ and $l$ reflect the approximate separability of the associated semiclassical wavefunctions in the local coordinates parallel and perpendicular to the periodic orbit. Nodal surfaces along the orbit are described by $n$, while $\left(k, \gamma_{1}\right)$ and $\left(l, \gamma_{2}\right)$ represent excitations perpendicular to the orbit. The number of nodes in the bending degree of freedom is given by $k$, whereas $l$ counts the number of nodes perpendicular to the orbit but preserving collinearity. The quantum number $n$ is not limited, but the number of possible transversal nodes, $k, l$, depend on $n$. The total number of states associated with the stability island is proportional to its phase space volume which according to equations (2) scales with $(-E)^{-3 / 2}$.

Re-ordering of equation (3) with respect to energy yields a Rydberg series of resonances converging to the double ionization threshold (Richter and Wintgen 1990a, 
1991)

$$
E_{n k l}=-\frac{S^{\mathrm{sc} 2}}{\left(n+\frac{1}{2}+2\left(k+\frac{1}{2}\right) \gamma_{1}+\left(l+\frac{1}{2}\right) \gamma_{2}\right)^{2}}
$$

Before applying (3) or (4) blindly, one should realize the inherent approximations and restrictions of these formulae. For integrable systems the equations actually represent an approximation of the torus (or EBK) quantization procedure, where the actions of the irreducible circuits on the tori are quantized separately (Miller 1975). Here these actions are approximated harmonically through the properties of the fixed point (periodic orbit) in the centre of the elliptic island. The advantage of such an approach is that it can also be applied to non-integrable systems, as long as the elliptic island surrounding the periodic orbit is large enough to support many eigenstates, i.e. its

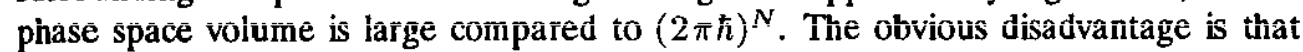
we cannot expect the approximation to be of good quality if the phase space volume of the island is small, or if the phase space structure (e.g. non-elliptic deformations of the tori) varies strongly over small phase space distances. However, due to the scaling properties for the present system such restrictions limit the applicability only to transversal excitations $k, l$, but not to $n$.

Note that the triple (i.e. containing three quantal contributions) Rydberg formula (4) yields real energies. In the lowest semiclassical approximation presented here the wavefunctions are square integrable and represent bound states. These states can autoionize semiclassically by dynamical tunnelling (Davis and Heller 1981), but the decay widths for such processes decrease exponentially with the nodal excitation along the orbit. Formula (4) applies to both symmetrical and antisymmetrical states of electron exchange (i.e. to the spectroscopic ${ }^{2 S+1} \mathrm{~L}^{\pi}={ }^{1} \mathrm{~S}^{\mathrm{e}}$ and ${ }^{3} \mathrm{~S}^{\mathrm{e}}$ series). Again, the exchange energies vanish exponentially and their precise determination is beyond the scope of the lowest order semiclassical treatment. All these semiclassical predictions will be compared with (numerically) exact quantum mechanical results in section 5 .

\section{Adiabatic approximation}

\subsection{The adiabatic Schrödinger equation}

A striking property of the classical periodic orbit of figure 1 is the large differences in the electronic velocities. Indeed, the action $S_{2}=\oint p_{2} \mathrm{~d} q_{2}=\int_{0}^{T} p_{2}^{2} \mathrm{~d} t$ of the inner electron is about $2 \times 10^{4}$ times larger than the action $S_{1}$ of the outer electron. This indicates that an adiabatic quantum mechanical treatment similar to the BornOppenheimer (BO) approximation in molecular physics should be applicable. The adiabatic coordinate is then the axis $r_{1}$ hetween the nucleus and the outer electron.

To express the three-body Coulomb problem in molecular coordinates we introduce a coordinate $r$ which determines the position of the inner electron relative to the geometrical centre between the nucleus and the outer electron,

$$
\boldsymbol{R}=\boldsymbol{r}_{1} \quad \boldsymbol{r}=\boldsymbol{r}_{2}-\boldsymbol{R} / 2 .
$$

Neglecting spin-orbit effects, the coupling of the electronic momenta and a further mass polarization term, which results from the choice of the geometrical centre as reference point for the inner electron, the Hamiltonian becomes (Pack and 
Hirschfelder 1968)

$$
H=-\frac{1}{2} \nabla_{R}^{2}-\frac{Z}{R}+h_{\mathrm{BO}}
$$

with the Born-Oppenheimer Hamiltonian $h_{\mathrm{BO}}$ for the inner electron,

$$
h_{\mathrm{BO}}=-\frac{1}{2} \nabla_{r}^{2}-\frac{Z}{|\boldsymbol{R} / 2+r|}+\frac{1}{|\boldsymbol{R} / 2-\boldsymbol{r}|} .
$$

The conserved total angular momentum $L$ is decomposed as

$$
L=L_{R}+l
$$

where $L_{R}=-\mathrm{i} R \times \nabla_{R}$ and $l$ is the angular momentum of the inner electron. The kinetic energy operator $\nabla_{R}^{2}$ can be divided into radial and angular parts

$$
-\frac{1}{2} \nabla_{R}^{2}=\frac{p_{R}^{2}}{2}+\frac{L_{\mathrm{R}}^{2}}{2 R^{2}}
$$

The Euler angles $\psi, \theta$ and $\varphi$ describe the rotation from a space-fixed to a bodyfixed frame (with the $z$-axis along $\boldsymbol{R}$ ) and are used to express $\boldsymbol{R}$ by the coordinates $\psi, \theta$ and $R$. The position vector $r$ is specified by two coordinates (in the following called $\lambda, \mu$ ) determining the position of the inner electron in the body-fixed $(\boldsymbol{R}, \boldsymbol{r})$ plane and by the azimuthal angle $\varphi$ with respect to $\boldsymbol{R}$. Following Feagin and Briggs (1988) the spatial wavefunction $\Psi_{L M}$ for total angular momentum $L$ and space-fixed projection $M$ can be expanded as

$\Psi_{L M}(\boldsymbol{r}, \boldsymbol{R})=\sum_{-L \leqslant m \leqslant L} \sum_{\{i\}} D_{M m}^{L}{ }^{*}(\psi, \theta, \varphi) \frac{1}{R} f_{i m}^{L}(R) \phi_{i m}(\lambda, \mu ; R)$.

The rigid top wavefunctions $D_{M m}^{L}$ describe the overall rotation of the three-body complex with total angular momentum $L$. They are eigenfunctions of $\boldsymbol{L}^{2}$ with eigenvalue $L(L+1)$ and eigenfunctions of $L_{z}$ with eigenvalues $M$ and $m$ in the spaceand body-fixed frames, respectively. They are also eigenfunctions of $l_{z}$ with eigenvalues $m$, since the $z$-component of $L_{R}$ is zero $\left(L_{R} \cdot R \equiv 0\right)$. The functions $\phi_{i m}$ are molecular orbital (MO) wavefunctions describing the motion of the inner electron $(r)$ for fixed $R$. The indices $i$ denote the set of quantum numbers for the two remaining degrees of freedom $\lambda, \mu$ of $r$. The wavefunctions $(1 / R) f_{i m}^{L}(R)$ describe the (slow) vibration of the outer electron. In the following we focus on states with $L=0$. Then $L=M=m=0$ and the total spatial wavefunction reduces to (dropping the indices $L, M, m)$

$$
\Psi(r, R)=\sum_{\{i\}} \frac{1}{R} f_{i}(R) \phi_{i}(\lambda, \mu ; R)
$$

The disappearance of the rotational wavefunction $D_{M m}^{L}\left(D_{00}^{0}\right.$ is a constant function) reflects the fact that the motion now takes place in a space-fixed plane.

The wavefunction (11) does not take into account the exchange symmetry of the identical particles, i.e. the electrons (Pauli principle). Effects due to (anti-) symmetrizing the wavefunction are neglected in this adiabatic approach. After integration over 
the inner coordinates $\lambda, \mu$, the Schrödinger equation for the Hamiltonian (6) together with equation (9) and the expansion (10) reduces to the set of coupled channel equations (Feagin and Briggs 1988)

$$
\begin{aligned}
\left(-\frac{1}{2} \frac{\partial^{2}}{\partial R^{2}}\right. & \left.-\frac{Z}{R}+\varepsilon_{i}(R)+\left\langle\phi_{i}\left|-\frac{1}{2} \frac{\partial^{2}}{\partial R^{2}}+\frac{L_{R}^{2}}{2 R^{2}}\right| \phi_{i}\right\rangle-E\right) f_{i}(R) \\
& =-\sum_{i \neq j}\left(\left\langle\phi_{i}\left|-\frac{1}{2} \frac{\partial^{2}}{\partial R^{2}}+\frac{l^{2}}{2 R^{2}}\right| \phi_{j}\right\rangle+\left\langle\phi_{i}\left|\frac{1}{R} \frac{\partial}{\partial R}\right| \phi_{j}\right\rangle \frac{\partial}{\partial R}\right) f_{j}(R)
\end{aligned}
$$

where $\varepsilon_{i}(R)$ is the Bo energy,

$$
h_{\mathrm{BO}}\left|\phi_{i}(R)\right\rangle=\varepsilon_{i}(R)\left|\phi_{i}(R)\right\rangle .
$$

For $L=0$ there is no rotational (Coriolis) coupling; the non-diagonal parts on the right-hand side of equation (12) originate from the radial coupling only. In the singlechannel adiabatic approximation used here these coupling elements are neglected. We will discuss their influence later on.

\subsection{The adiabatic potentials}

As a first step the Schrödinger equation (13) for the inner electron has to be solved for fixed $R$ to get the energy surface $\varepsilon_{i}(R)$. The Bo energies include the averaged electron-electron interaction. The energy surface contributes to the effective potential in the channel equations (12) for the outer electron. Finally, the solution of equation (12) yields the total energy of the three-body Coulomb complex.

As is well known from molecular physics (Helfrich 1972, Slater 1977) the Schrödinger equation for an electron moving in the field of two fixed charged particles is separable in prolate spheroidal coordinates $\lambda, \mu$, which for our coordinates (5) read

$$
\lambda=\frac{r_{2}+r_{12}}{R} \quad \mu=\frac{r_{2}-r_{12}}{R}
$$

with $r_{2}=|\boldsymbol{r}+\boldsymbol{R} / 2|$ and the inter-electron distance $r_{12}=|\boldsymbol{r}-\boldsymbol{R} / 2|$. Thus the Mo wavefunctions $\phi_{i}$ for the inner electron, which are eigenfunctions of the MO Hamiltonian $h_{\text {BO }}$ (equation (7)), separate in prolate spheroidal coordinates, $\phi_{i}(\lambda, \mu)=\xi_{n_{\lambda}}(\lambda) \eta_{n_{\mu}}(\mu)$. The function $\xi_{n_{\lambda}}(\lambda)$ has elliptical nodal surfaces in configuration space with the nucleus and the outer electron as foci. The function $\eta_{n_{\mu}}(\mu)$ possesses a corresponding hyperbolic nodal structure (Rost and Briggs 1989). The nodal quantum numbers $n_{\lambda}$ and $n_{\mu}$ are conserved for arbitrary parameter $R$. In the limit of large $R$ (equivalent to the separated atom limit in molecular physics) the quantum numbers $n_{\lambda}$ and $n_{\mu}$ coincide with parabolic coordinate quantum numbers $n_{1}$ and $n_{2}$ (Rost and Briggs 1991). The effect of the outer electron is then to produce an electric field which is nearly constant over the spatial range experienced by the inner electron. Thus the inner wavefunctions merely become Stark-like states of the remaining $\mathrm{He}^{+}$ion. 
The BO eigenvalues $\varepsilon_{n_{\lambda} n_{\mu}}(R)$ also yield the correct fragmentation limit: if the outer electron is removed, $(R \rightarrow \infty)$, the two-centre Hamiltonian (7) becomes a hydrogen-like Hamiltonian for the inner electron,

$$
h_{\mathrm{BO}}(R \rightarrow \infty) \rightarrow-\frac{1}{2} \nabla_{r_{2}}^{2}-\frac{Z}{r_{2}}
$$

with eigenvalues $\varepsilon(R \rightarrow \infty)=-Z^{2} / 2 N^{2}$.

The quantum analogue of the asymmetric (collinear) classical configuration of figure 1 consists of an inner electron in a molecular type state of maximal polarization along the axis $\boldsymbol{R}$. For a principal hydrogenic quantum number $N=n_{\lambda}+n_{\mu}+|m|+1$ of the inner electron this implies $n_{\lambda}=0$ (minimal off-radial excitation) and $n_{\mu}=$ $N-1$ (maximal number of nodes along $\boldsymbol{R}$ ).

There are several procedures available to solve the two-centre problem for the inner electron numerically (Power 1973, Salin 1978). Here we used a method of continued fractions which is described in Solov'ev (1981). We will mainly focus on results for helium $(Z=2)$; calculations for other atoms (ions) of the isoelectronic sequence are discussed briefly in section 7 .

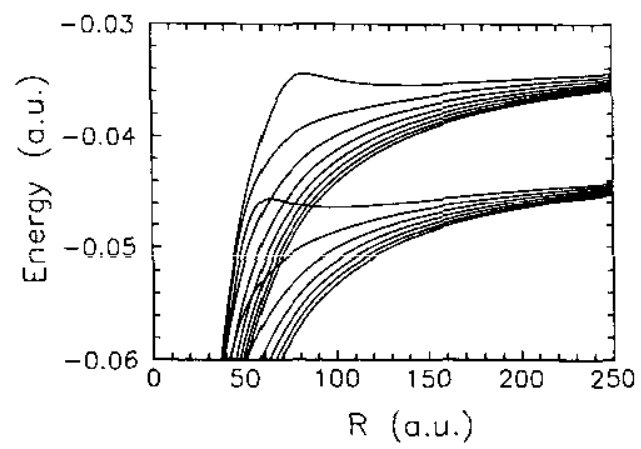

Figure 3. Born-Oppenheimer potential surfaces for the mo states belonging to the $N=7,8$-manifold.

Figure 3 shows the series of Bo potential curves

$$
U_{n_{\lambda} n_{\mu}}(R)=\varepsilon_{n_{\lambda} n_{\mu}}(R)-Z / R
$$

for ail mo states belonging to the $N=\overline{7}, \hat{8}$ manifolds. The potential curves for fixed $N$ are labelled by $n_{\lambda}$ and $n_{\mu}=N-n_{\lambda}-1$ with $n_{\lambda}$ running from zero (upper curve) to $N-1$ (lower curve). All curves other than the uppermost of each manifold are purely attractive, corresponding to the nuclear Coulomb field at short distances and the residual $\mathrm{He}^{+}$ionic field at large distances. (Note that for low total angular momentum the inclusion of a centrifugal barrier gives rise to a repulsive potential contribution only for $R \ll 50$ au for the cases shown in figure 3.) The uppermost curve, in which the fast inner electron is maximally polarized along the inter-electronic axis, develops a secondary potential well, around $R \sim 100$ au in figure 3 . The origin of this outer well has been explained already by Richter et al (1991). The potential barrier corresponds to the dynamical barrier appearing in the classical solution and prevents the outer electron from penetrating the nucleus. It is this outer minimum 
which leads to bounded vibrational motion, with oscillator-like eigenfunctions for the outer electron. These resonant states, localized in the outer potential well of the $n_{\mu}=N-1$ curve, are the main subject of this paper. The character of the infinity of resonances with energies high above the potential barrier of this curve is well known and is decided by the long-range $\mathrm{e}^{-}-\mathrm{He}^{+}\left(N, n_{\lambda}, n_{\mu}, m\right)$ potential and will not be discussed here.

For large $R$ each mo manifold merges into a Stark multiplet as was discussed above and which can be seen explicitly in figure 3 . The degree of polarization of the inner electron depends on $N$ and $n_{\lambda}$. Only those mo curves in figure 3 which are related asymptotically to the Stark states of maximal orientation along the 'field axis' $R$ show a potential well. For $n_{\lambda}=0$ and starting with $N=6$ the Bo potentials show a minimum sufficiently pronounced to allow for quantized vibrational states. Below this value the off-radial extent of the inner electron Mo wavefunction is too large. For the same reason wavefunctions with one or more nodal excitations perpendicular to $\boldsymbol{R}\left(n_{\lambda} \neq 0\right)$ do not support a potential minimum in figure 3 .

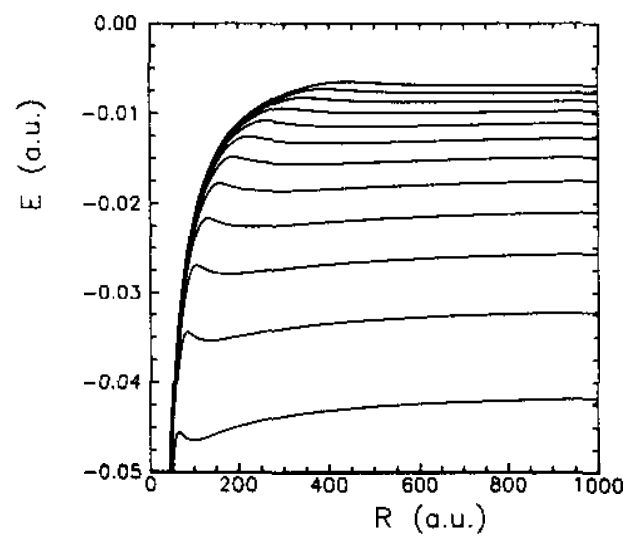

Figure 4. Upper Bo potentials $\left(n_{\lambda}=0\right)$ of the manifolds $N=7$ to $N=16$.

In figure 4 we show all upper Bo potential curves $\left(n_{\lambda}=0\right)$ with $n_{\mu}=N-1$ (the number of nodes along $R$ ) varying from 6 to 17 . It is not the depth but the radial extent of the potential well which increases as $n_{\mu}$ increases. The potential curves seem to flatten dramatically for larger $N$, but this optical illusion is related to the absolute $R$ scale used in the figure. To see this we derive an approximate scaling property for the BO potentials by exploiting the usual Coulombic scaling for energies and lengths which scale with $1 / N^{2}$ and $N^{2}$, respectively. The transformation

$$
\begin{aligned}
& U_{0, N-1} \rightarrow U_{0, N-1}=\frac{3 N(N-1)}{4}\left(U_{0, N-1}+\frac{2}{N^{2}}\right) \\
& R \rightarrow \mathcal{R}=\frac{4}{3 N(N-1)} R
\end{aligned}
$$

(which is the special case for helium of a more general $Z$-dependent scaling technique discussed in section 7 ) allows for a compact representation of the Bo potentials. The scaled potentials $U$ all converge to the common fragmentation energy zero. This is shown in figure 5, where scaled potential surfaces from figure 4 are drawn. The shape 
of the scaled potentials is only weakly $N$-dependent. The maxima of the potential barriers are all localized around $\mathcal{R}=2$ and become more pronounced with increasing $N$. This behaviour is expected from the increasing polarizability of the inner electron.

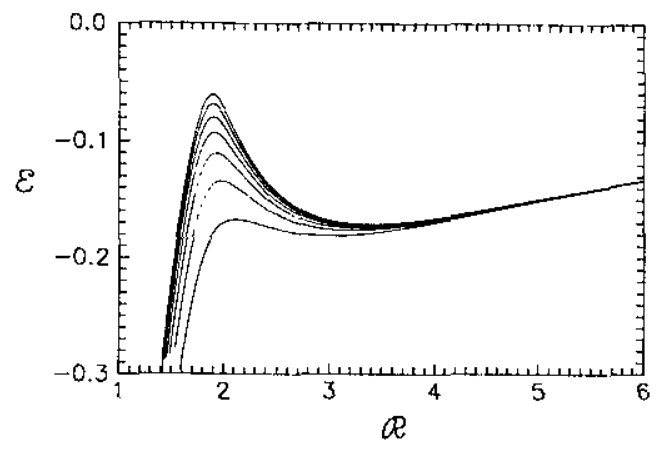

Figure 5. Scaled upper $B O$ potentials for $N=6,8,10,12,14,16$ (from bottom to top), see equation (17).

The Bohr-Sommerfeld ( $\overline{\mathrm{WK}} \overline{\mathrm{B}})$ quantization

$$
S=\int_{R_{\min }}^{R_{\mathrm{mix}}} \sqrt{2(E-U(R))} \mathrm{d} R=\ell+\frac{1}{2}
$$

of the motion between the turning points $R_{\min }$ and $R_{\max }$ defines a new vibrational quantum number $\ell$. In the adiabatic approach a doutoly excited two-êlectron state is now characterized by the molecular quantum numbers $n_{\lambda}, n_{\mu}$, the vibrational quantum number $\ell$, and the (exact) quantum numbers of angular momentum $L=$ $M=m=0$. The quantization condition (18) yields the energies and the number of vibrational eigenstates in each potential well. The potential well for $N=7$, for example, contains only two vibrational states with a spacing of $\Delta E=0.00078$ au $(\approx 21 \mathrm{meV}$ ), while the adiabatic $N=18$ potential already supports about 20 states with a mean spacing of the order of $1 \mathrm{meV}$. The number of vibrational states in the adiabatic approximation can be estimated by exploiting the (approximate) scaling property (17) of the adiabatic potentials. For large $N$, scaling of the quantization condition (18) leads to

$$
\sqrt{\frac{3}{2}}\left(N-\frac{1}{2}\right) \mathcal{S}^{(N)}(\mathcal{E})=\hat{\ell}+\frac{1}{2}
$$

with

$$
\mathcal{S}^{(N)}(\mathcal{E})=\int_{\mathcal{R}_{\text {min }}}^{\mathcal{R}_{\text {max }}} \sqrt{2\left(\mathcal{E}-U_{0, N-1}(\mathcal{R})\right)} \mathrm{d} \mathcal{R}
$$

The number of vibrational states $\ell_{\max }$ is proportional to $(N-1 / 2) \mathcal{S}_{N}\left(\mathcal{E}_{\max }\right)$, where $\mathcal{E}_{\text {max }}$ is the scaled energy of the potential maximum.

Since $\mathcal{S}^{(N)}$ grows monotonically with $N$ (this can be derived numerically and it is also obvious from figure 5) equation (19) shows that the number $\ell_{\max }$ of vibrational eigenstates increases slightly faster than $N$. However, it is not clear whether the 
single-channel adiabatic approach is still meaningful when the vibrational excitation $\ell$ is of the order of the principal quantum number $N$ of the inner electron. In fact, the neglect of the radial coupling matrix elements in the coupled channel equation (12) is justified only for small radial velocities. It is for this reason that we focus only on the states localized in the shallow outer potential well, even though each potential curve supports, in principle, a Rydberg series due to the long-ranged Coulomb tail of the potential.

The occurrence of minima in the BO potentials is not restricted to MO states with $n_{\lambda}=0$. If $N$ is large enough (i.e. $N \geqslant 16$ in the case of helium), the polarization of an inner-electron state with one off-radial node $\left(n_{\lambda}=1\right)$ is strong enough to produce a potential well in the adiabatic potential. Figure 6 shows both the $n_{\lambda}=0$ and the $n_{\lambda}=1$ potentials of each $N$-manifold $(N=14$ to 18$)$ from which a build-up of the minima for $n_{\lambda}=1$ can be seen. As long as a sufficient ratio $n_{\mu} / N$ of excitation nodes along $\boldsymbol{R}$ is preserved, the adiabatic potentials will exhibit a secondary minimum allowing for dynamical spatial localization of the outer electron.

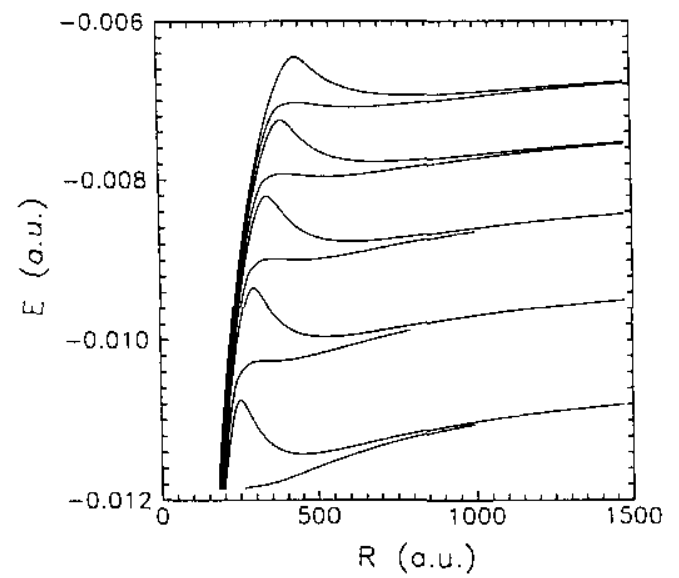

Figure 6. The highest two BO potentials $\left(n_{\lambda}=0,1\right)$ of the manifolds $N=14$ to $N=18$.

Hitherto the discussion has been based on the 'static' Bo potential $U=$ $\left\langle\phi_{i}\left|h_{\mathrm{BO}}\right| \phi_{i}\right\rangle-Z / R$ of the radial equation (12) ignoring the diagonal elements $\left\langle\phi_{i}\left|-\partial^{2} / \partial R^{2}+L_{R}^{2} / R^{2}\right| \phi_{i}\right\rangle$ which contain the centrifugal barrier of the outer electron. Rewriting the diagonal element as $\left\langle\phi_{i}\left|\frac{1}{2} p_{R}^{2}\right| \phi_{i}\right\rangle$ (see equation (9)) it is evident from the analysis of the classical motion, that it should be small compared to the inner electronic kinetic energy part $\left\langle\phi_{i}\left|\frac{1}{2} p_{r}^{2}\right| \phi_{i}\right\rangle$ of the Hamiltonian $h_{\mathrm{BO}}$. The centrifugal correction $\left\langle\phi_{i}\left|L_{R}^{2} /\left(2 R^{2}\right)\right| \phi_{i}\right\rangle$ can be considered analytically if we approximate the MO functions $\phi_{n_{\lambda} n_{\mu}}$ by parabolic eigenfunctions $\phi_{n_{1} n_{2} m}$ of the $\mathrm{He}^{+}$ion. For $L=0=L_{R}+l$ the centrifugal term is given by $\left\langle\phi_{n_{1} n_{2} m}\left|l^{2}\right| \phi_{n_{1} n_{2} m}\right\rangle /\left(2 R^{2}\right)$. An expansion of the parabolic states for $n_{1}=N-1, n_{2}=m=0$ into the spherical eigenfunction basis of $l^{2}$ yields the simple result

$$
\left\langle\phi_{N-1,0,0}\left|l^{2}\right| \phi_{N-1,0,0}\right\rangle=(N-1) .
$$

Thus a centrifugal term $(N-1) /\left(2 R^{2}\right)$ is added to the Bo potentials (16) representing the rotational motion of the outer electron. However, this only amounts to 
a very slight change in the potential surfaces. The effect of the centrifugal diagonal correction on the energies of the two-electron states is discussed in section 5 .

For non-vanishing total angular momentum $L$ the entire rotational energy of the three-body system leads to an additional raising of the adiabatic potential barrier and to a shift of the minima towards larger $R$. However, again the overall structure (in particular the appearance of a secondary outer minimum) is not affected.

The adiabatic analysis presented here uses a single-channel approximation ignoring the non-diagonal terms of the coupled channel equation (12). In the molecular case $\left(\mathrm{H}_{2}^{+}\right)$these elements are small due to the inertia of the internuclear axis. However, in the two-electron problem there exist no obvious geometrical or kinematical reason to neglect them in general. As long as the MO potentials are energetically well separated off-diagonal couplings should be small (they are inversely proportional to the energy difference of corresponding levels, which is a consequence of the Heliman-Feynman theorem). Nevertheless, in regions of avoided crossings of adiabatic potential curves non-diagonal couplings can become important. In our approach we are interested in the coupling of one upper BO potential with all the other adiabatic channels. An adiabatic treatment which includes such diagonal and non-diagonal corrections-shows only very small avoided crossings between the BO curves near the potential minima (Thürwächter 1992). This supports the applicability of our simplified single-channel adiabatic approximation at least for states of small vibrational excitation $\ell$.

Calculated energies for doubly excited states obtained within this single-channel adiabatic approximation are compared with exact results in section 5 .

\section{Non-approximate quantum solutions}

In this section we describe our $a b$ initio method to solve (with nine-figure accuracy) the Schrödinger equation for highly doubly excited electron states. A full solution of the Schrödinger equation for highly doubly excited states is a non-trivial problem. - Here we use a transformation of the Schrödinger equation into perimetric coordinates (James and Coolidge 1937). Even though this coordinate set had already been used in numerical ground-state calculations as early as 1958 (Pekeris), its power and simplicity for the calculations of highly excited states has not been fully recognized in the past. We obtain resonance positions and resonance widths on small computers within nearmachine precision even for highly excited states (Richter and Wintgen 1991). This allows us to check very accurately the predictions of the classical, semiclassical and adiabatic approximations described in sections 2 and 3.

Using perimetric coordinates defined as

$$
\begin{aligned}
& x=r_{1}+r_{2}-r_{12} \\
& y=r_{1}-r_{2}+r_{12} \quad x, y, z \geqslant 0 \\
& z=-r_{1}+r_{2}+r_{12}
\end{aligned}
$$

the Hamiltonian (1) for $L=0$ reads (with $\left(x_{1}, x_{2}, x_{3}\right)$ defined as $(x, y, z)$ )

$H=\frac{1}{(x+y)(x+z)(y+z)} \sum_{i, j=1}^{3} \frac{\partial}{\partial x_{i}} P_{i j}^{(3)}(x, y, z) \frac{\partial}{\partial x_{j}}-\frac{Z}{x+y}-\frac{Z}{x+z}+\frac{1}{y+z}$. 
The $P_{i j}^{(3)}$ are polynomials of degree 3 and can be found, for example, in Frost et al (1964) and Zhen (1990). We expand each degree of freedom in a complete Sturmian basis set and (anti-) symmetrize the product functions (the electron exchange corresponds to an exchange of the perimetric coordinates $y$ and $z$ ),

$$
\Phi_{n m k}^{ \pm}(x, y, z)=\phi_{n}(\alpha x)\left[\phi_{m}(\beta y) \phi_{k}(\gamma z) \pm \phi_{k}(\gamma y) \phi_{m}(\beta z)\right]
$$

with $\phi_{n}(u)$ defined by

$$
\phi_{n}(u)=L_{n}(u) \mathrm{e}^{-u / 2}
$$

and $L_{n}(u)$ are the Laguerre polynomials. The volume element

$$
\mathrm{d} V=\frac{1}{32}(x+y)(x+z)(y+z) \mathrm{d} x \mathrm{~d} y \mathrm{~d} z
$$

cancels the singularities in the kinetic energy and in the potential terms if matrix elements are calculated. All the matrix elements are of simple analytical form. Their calculation requires mostly integer arithmetic and is fast and accurate. In addition, selection rules guarantee that most of them vanish. The resulting matrix equation has a banded, sparse structure and allows for efficient diagonalization.

We use the method of complex rotation (Reinhardt 1982, Junker 1982, Ho 1983, Delande et al 1991) to calculate accurate positions and decay widths of the autoionizing two-electron resonances. Writing $\alpha$ and $\gamma$ as $\alpha=a \beta, \gamma=c \beta$, the Schrödinger equation has the particular form of the matrix equation

$$
\left(\beta^{2} \hat{\mathbf{T}}+\beta \hat{\mathbf{V}}-E \hat{\mathbf{N}}\right) \Psi=0 .
$$

The scaled matrices $\hat{\mathbf{T}}, \hat{\mathbf{V}}, \hat{\mathbf{N}}$ of the kinetic energy $T$, potential $V$ and the unit operator $N$ are symmetric and depend on $a$ and $c$ but not on $\beta$.

For the present calculations we used $c=1$ (which increases the sparseness of the matrices considerably) and $a$ real (a typical value is $a=2$ ). The scaled matrices are then all real symmetric, sparse, banded and independent of the scale parameter $\beta$, which is, however, complex.

We solved the complex symmetric matrix equation (27) with an inverse iteration method using an $L D L^{\mathrm{T}}$ decomposition of the matrix (27) to obtain complex eigenvalues and eigenvectors. For each eigenfunction we used an iterative algorithmn to optimize the scale parameter $\beta$ such that the complex energy becomes stationary, $\partial E / \partial \beta=0$. The wavefunction then fulfils the complex virial theorem (Ho 1983) $-2\langle T\rangle=\langle V\rangle=2 E$. We checked the convergence of the eigenvalues by increasing the basis size and by checking the expectation values of the partial derivative $\partial E / \partial a$, which vanishes for an exact wavefunction. We varied the basis size by systematically increasing the total number of nodes $N=n+m+k$ of the basis functions (24). Rypical values used were $N=10, \ldots, 42$ (corresponding to matrix dimensions (bandwidths) ranging from $144(38)$ to $7337(519)$ ), for which we get $|\partial E / \partial a|<10^{-10}$ and an accuracy of the complex energies of 10 or more significant digits. Numerical values for expectation values are only slightly less converged. We used certain expectation values to identify the proper states out of the vast number of resonances occurring. To give an estimate of the energy region covered by our calculations we note that doubly excited intra-shell resonances with $N$ ranging from 6 to 18 cover this energy region (we do not discuss here whether such states can still be classified in a meaningful way using intra-shell labels).

Results for the many resonances calculated but not discussed here will be presented elsewhere. 


\section{Results}

\subsection{Overview}

The application of the approaches described in sections $2-4$ can be summarized as follows.

(i) The semiclassical method predicts a triple Rydberg series of long-lived resonances converging to the double ionization threshold. The quantum numbers involved describe nodal excitations along the local coordinates of the frozen-planet periodic orbit, i.e. parallel $(n)$ and perpendicular to the orbit $(k, l)$. The associated wavefunctions separate approximately in these local coordinates.

(ii) The adiabatic treatment leads to the set of MO quantum numbers $n_{\mu}$ (number of nodes along the radial outer-electron vector $\boldsymbol{R}$ ), $n_{\lambda}$ (off-radial excitations) and $\ell$ (vibrational nodes of the outer electron). We will show below that there exists a one-to-one correspondence with the semiclassical set of quantum numbers $(n, k, l)$.

(iii) The full quantum calculations yield highly accurate resonance energies, decay widths and wavefunctions which do not rely on any underlying assumption or approximation.

We will first report on our results for the states of the 'principal series' $(n, k, l)=$ $(n, 0,0)$, i.e. all nodal cxcitations arc along the periodic orbit. We then discuss results for some 'satellite series' $(n, k, 0)$ and $(n, 0, l)$.

\subsection{The principal series $(n, 0,0)$}

5.2.1. Energies. In table 1 we summarize our results for the energies of the resonant states of the principal ${ }^{1} S^{e}$ and ${ }^{3} S^{e}$ series. The energies for $n=2,3$ of ${ }^{1} S^{e}$ symmetry had already been calculated by Ho (1986), but with less accuracy. The table gives the numerically converged results of the quantum calculations as well as the approximate values $E_{\mathrm{scl}}$ predicted by the simple semiclassical formula (4) and the approximate values $E_{\mathrm{BO}}$ obtained by solving the single-channel equations with the adiabatic potentials supporting a secondary minimum $(n>5)$. States with $n \ll 6$ may be called precursors, since they possess a character which transforms smoothly into that of the higher excited members of the series. The adiabatic potentials for the precursor states only show a more or less pronounced plateau at large distances but not a secondary minimum. Particular properties of the states to be discussed in the following subsections are typically less pronounced for the precursor states.

As can be seen from table 1 the simple semiclassical formula is superior to the more elaborate adiabatic calculations in predicting the quantum energies accurately. The errors of the semiclassical energies are below $1 \%$ for all resonances (except the low-lying $n=2$ state of the ${ }^{1} \mathrm{~S}^{\mathrm{e}}$ subspace, where the error is about $3 \%$ ) and below $0.1 \%$ for states with $n>7$. In the derivation of the WKB-type formula (3) all expressions are consistently expanded to leading order in $\hbar$. The intrinsic error of the lowest-order semiclassical approach is then of the order of $\hbar^{2}$, which in the present context is equivaient to a decreasing error for the effective quantum number (or equivalently the quantum defect) proportional to $1 / n$. In figure 7 we plot the semiclassical error for the quantum defect-like quantity $\mu_{n}$,

$$
\mu_{n}=N_{\mathrm{eff}}-n
$$


Table 1. Energies of the $(n, 0,0)$ frozen-planet configurations obtained by full quantum solutions (singlet and triplet states respectively), the semiclassical triple Rydberg formula (4) $\left(E_{\mathrm{scl}}\right)$, an improved semiclassical treatment $\left(E_{\mathrm{scl}}^{\mathrm{corr}}\right)$, the single-channel adiabatic approximation $\left(E_{\mathrm{BO}}\right)$, and the adiabatic approximation including diagonal corrections as described in the text $\left(E_{\mathrm{BO}}^{\mathrm{cor} r}\right)$. The numbers are truncated, not rounded. The error estimate is \pm 1 in the last digit.

\begin{tabular}{rllllll}
\hline$n$ & $-E\left({ }^{1} \mathrm{~S}^{\mathrm{e}}\right)$ & $-E\left({ }^{3} \mathrm{~S}^{\mathrm{e}}\right)$ & $E_{\text {scl }}$ & $E_{\text {scl }}^{\text {corr }}$ & $E_{\mathrm{BO}}$ & $E_{\mathrm{BO}}^{\text {corr }}$ \\
\hline 2 & 0.257371609 & 0.249964615 & 0.247923 & 0.250980 & - & - \\
3 & 0.141064156 & 0.140088483 & 0.139351 & 0.140216 & - & - \\
4 & 0.089570804 & 0.089467826 & 0.089144 & 0.089472 & - & - \\
5 & 0.062053558 & 0.062041278 & 0.061887 & 0.062038 & - & $\mathbf{0 . 0 6 2 3 0 3}$ \\
6 & $\mathbf{0 . 0 4 5 5 3 8 6 6 7}$ & 0.045539242 & 0.045458 & 0.045537 & 0.045956 & $\mathbf{0 . 0 4 5 6 8 7}$ \\
7 & $\mathbf{0 . 0 3 4 8 4 2 6 4 2}$ & 0.034843857 & 0.034798 & 0.034843 & 0.035109 & 0.034939 \\
8 & 0.027517599 & 0.027519289 & 0.027491 & 0.027519 & 0.027612 & 0.027584 \\
9 & 0.022284587 & 0.022283665 & 0.022265 & 0.022284 & 0.022413 & 0.022330 \\
10 & 0.018411985 & 0.018411896 & 0.018400 & 0.018412 & 0.018507 & 0.018446 \\
11 & 0.015468259 & 0.015468265 & 0.015460 & 0.015468 & 0.015541 & 0.015494 \\
12 & 0.013178121 & 0.013178140 & 0.013172 & 0.013178 & 0.013235 & 0.013199 \\
13 & 0.011361442 & 0.011361444 & 0.011357 & 0.011361 & 0.011406 & 0.011378 \\
14 & 0.009896121 & 0.009896120 & 0.009893 & 0.009896 & 0.009932 & 0.009909 \\
\hline
\end{tabular}

where the effective quantum number $N_{\text {eff }}$ is defined as the denominator in equation (4), i.e.

$$
N_{\text {eff }}=S^{\mathrm{sc}} / \sqrt{-E_{n k l}}
$$

where $E_{n k l}$ are the quantum eigenvalues. From figure 7 we deduce the leading term of the semiclassical error in the quantum defect $\mu_{n}$ to be $-0.0366 / n$. Inclusion of this higher order term in the triple Rydberg formula (4) yields the improved energy values $E_{\mathrm{scl}}^{\mathrm{corr}}$ of table 1. The higher-order semiclassical Rydberg formula reproduces the exact quantum calculations perfectly well and can be used to predict even higherlying members of the principal series to an accuracy of several significant digits.

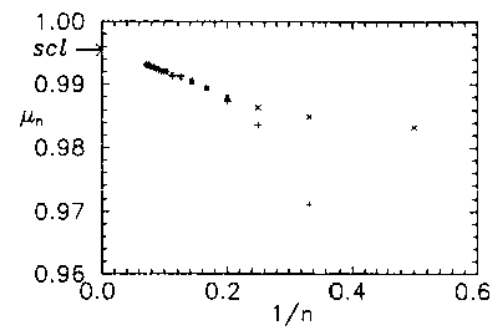

Figure 7. Quantum defect $\mu_{n}$ as defined by equation (28). Both parity classes, ${ }^{1} S^{e}(+)$ and ${ }^{3} S^{e}$ $(x)$, are shown. The semiclassical linit is marked with an arrow.

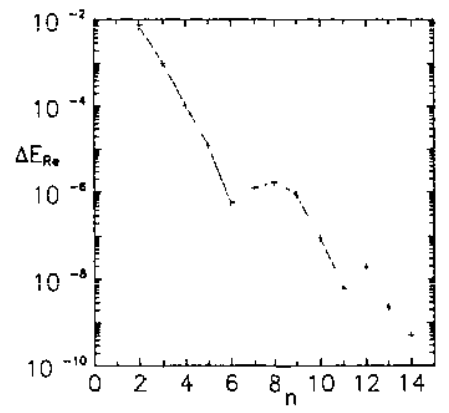

Figure 8. Absolute energy differences of singlet and triplet $(n, 0,0)$ states on a logarithmic scale.

Such an improved Rydberg formula still neglects particle exchange effects, but as discussed in section 2 semiclassical considerations suggest that the energy splitting 
due to electron exchange is suppressed exponentially with increasing nodal excitation $n$. This is shown in figure 8 where we plot the absolute value of the energy splitting of our highly accurate quantum results on a logarithmic scale. Obviously, the general trend confirms the semiclassical estimate.

Column 6 of table 1 gives the energies $E_{\mathrm{BO}}$ of the vibrational eigenstates in the outer wells of the BO potentials shown in figure 4, while column 7 gives the improved results $E_{\mathrm{BO}}^{\text {carr }}$ by including the diagonal centrifugal correction terms as described in section 3. Even though inclusion of these terms improves the accuracy significantly, the adiabatic approach does not reach the high degree of agreement achieved between the semiclassical and the exact quantum results. Whereas the semiclassical formula reproduces the exact results of the coupled equations of quantum motion to greater and greater accuracy with increasing $n$, the single-channel adiabatic approximation presented here neglects parts of the full Hamiltonian (non-diagonal couplings and the radial parts of the diagonal elements in equation (12)) and cannot be expected to converge to the exact results. Inclusion of the neglected terms, however, would complicate the adiabatic approach tremendously.

5.2.2. Wavefunctions. Compared with energy eigenvalues, a direct examination of the nodal structure of the associated wavefunctions is a more stringent test of the different approximations. Figure $9(a)$ depicts the probability distribution of the $a b$ initio wavefunction for the $(6,0,0)$ state of the principal series in the $(z=0)$ plane of the perimetric coordinates, equation (23). This corresponds to the collinear arrangement of the electrons, i.e. $\Theta=0(\Theta$ represents the angle between the electronic radial vectors). The off-collinear part of the probability density, not shown here, decreases exponentially indicating a zero-point motion in the $\Theta$-bending degree of freedom. The zero-point motion is expressed by the assignment $k=0$ within local coordinate quantum numbers and by $n_{\lambda}=0$ within the Mo description respectively. The coordinate $r_{1}\left(r_{2}\right)$ denotes the radial distance of the outer (inner) electron. The outer electron probability is strongly localized in the region $r_{1} \approx 120$ au reflecting the dynamical localization of the 'frozen' electron. Note also the large differences in the radial extents $r_{i}$. The nodal excitations are all directed along the frozen-planet periodic orbit, which is a nearly straight line along the frozen-planet radius indicated by an arrow in the figure. Recalling the typical quadratic spacing of nodal lines in Coulombic systems, we achieve nearly constant nodal distances by using quadratically scaled axes as done in figure $9(d)$. The number of nodes along the orbit is $n=6$ in agreement with the semiclassical predictions. The wavefunction does not show any off-orbit excitations, which agrees with the semiclassical local coordinate classification $(n, k, l)=(6,0,0)$.

Within the Mo description the wavefunction is characterized as follows. The inner electron is maximally polarized along the nucleus-frozen-electron axis $R\left(n_{\lambda}=\right.$ $0, n_{\mu}=6$ ) while the outer electron is in its vibrational ground state $(\ell=0)$ of the effective potential well. Note the corresponding absence of nodal lines in $r_{1}$ for the wavefunction in figure $9(a)$. Thus the wavefunction of the principal series reveals the equivalence of the semiclassical local coordinate quantum number $n$ and the molecular-type quantum number $n_{\mu}$.

5.2.3. Expectation values. An investigation of classical and quantum mechanical expectation values completes the comparison of the quantum results with the quasiclassical approach. We define a classical expectation value $\left\langle A\left(\boldsymbol{r}_{1}, \boldsymbol{r}_{2}\right)\right\rangle_{\mathrm{cl}}$ as the time 

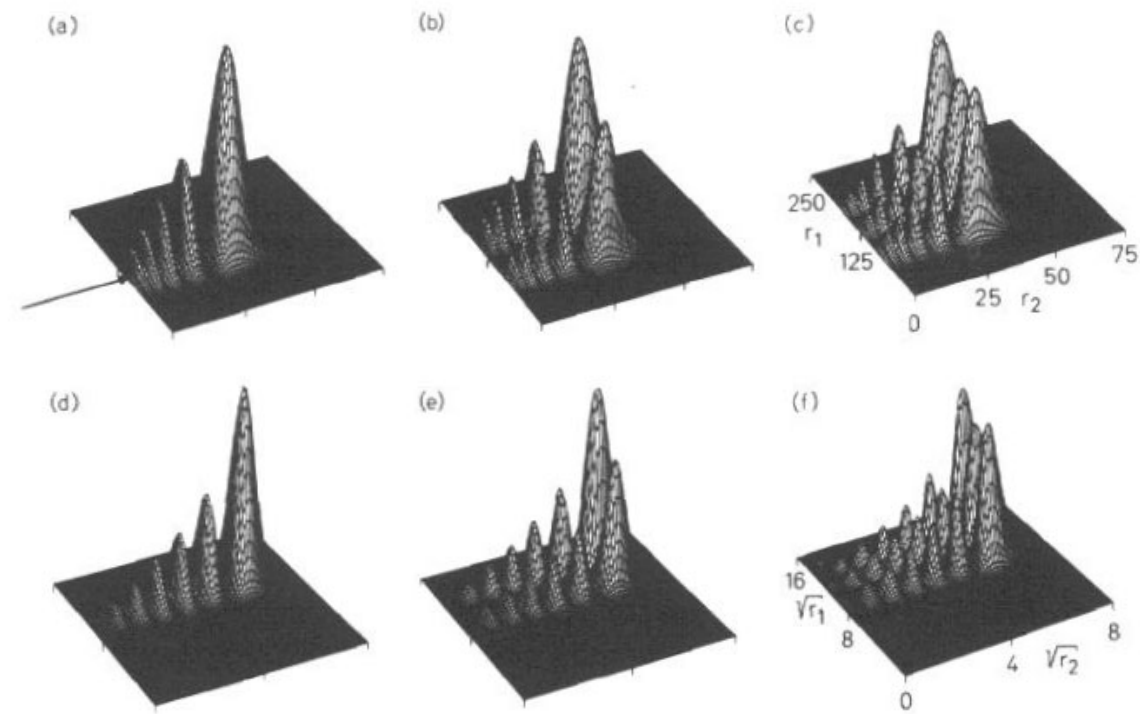

Figure 9. Conditional probability densities of $(n, 0, l)$ states for $n=6$. The angle $\Theta$ between $r_{1}$ and $r_{2}$ is fxed to $\Theta=0$. The axes have a linear (left part) and a quadratic scale (right part), respectively. Shown are $l=0(a)$ and $(d), l=1(b)$ and $(c)$, and $l=2(c)$ and $(f)$. Note the asymmetry in the scales of the axes. Only the parts $r_{1}>r_{2}$ are shown. The fult wavefunction is symmetric in $r_{1}$ and $r_{2}$.

average of $A\left(r_{1}, r_{2}\right)$ along the frozen-planet periodic orbit,

$$
\left\langle A\left(r_{1}, r_{2}\right)\right\rangle_{\mathrm{cl}}=\frac{1}{T} \int_{0}^{T} A\left(r_{1}(t), r_{2}(t)\right) \mathrm{d} t
$$

Expectation values such as $\langle\cos \Theta\rangle_{c l}$ and $\left\langle r_{12}\right\rangle_{c]}$, are invariant under electron exchange and can be compared directly with the associated quantal expectation values. For the hyperangle $\tan \alpha=r_{1} / r_{2}$, which measures the asymmetry of the electronic excitation, we use the classically symmetrized form $(\tan \alpha)_{e l}=\frac{1}{2}\left\langle r_{2} / r_{2}+r_{2} / r_{1}\right\rangle_{\mathrm{cl}}$ to account for the (anti-) symmetrization of the quantal wavefunctions. Correspondingly, we define the mean radial distance $r$ of the electrons classically by $\frac{1}{2}\left\langle r_{1}+r_{2}\right\rangle_{\text {cl }}$. Again, the classical mechanics calculations cannot account for (exponentially small) exchange effects. All the quantum expectation values are complex valued (as is the energy) and we only list the real part. However, for the selected expectation values the imaginary part is typically smaller by two orders of magnitude.

The results for the principal series are summarized in table 2 for $n=2$ to 14 . Only the quantum results for ${ }^{1} S^{e}$ resonances are listed, the quantum calculations for ${ }^{3} S^{e}$ states yield similar results. Column 2 contains the values of $(\cos \Theta)$, which measures the degree of orientation and angular correlation of the electrons. With increasing excitation $\langle\cos \Theta\rangle$ converges to the classical value $\langle\cos \Theta\rangle_{c \mid}=1$ of maximal polarization of the electrons along the collinear configuration where both electrons are on the same side of the nucleus. (tan $\alpha$ ) converges to the energy independent limit $\langle\tan \alpha\rangle_{\mathrm{cl}}=2.887$ of the classical configuration. In columns 4 to 7 the energy scaled results for $\left\langle r_{12}\right\rangle,\langle r\rangle,\left\langle 1 / r_{12}\right\rangle$, and $\left\langle p_{1} \cdot p_{2}\right\rangle$ are tabulated. Except for the mass polarization term $\left\langle p_{3} \cdot p_{2}\right\rangle$, the deviations between classical and quantum results are only of the order of a few per cent, although expectation values are typically more 
Table 2. Expectation values of the $(n, 0,0)$ frozen-planet states of ${ }^{1} \mathrm{~S}^{\mathbf{e}}$ symmetry. The classical expectation values are given in the last row under the entry $n=\infty$. For convenience, energy-dependent expectation values are scaled with an appropriate energy dependent factor acconding to the classical scaling laws (2).

\begin{tabular}{rllllll}
\hline$n$ & $\langle\cos \Theta\rangle$ & $\langle\tan \alpha\rangle$ & $\left\langle r_{12}\right\rangle E$ & $\left\langle r_{1}\right\rangle E$ & $\left\langle 1 / r_{12}\right\rangle / E$ & $\left\langle p_{1} \cdot p_{2}\right\rangle / E$ \\
\hline 2 & 0.351 & 1.823 & 3.279 & 2.534 & 0.4065 & 0.0810 \\
3 & 0.534 & 2.215 & 3.729 & 2.941 & 0.3365 & 0.0442 \\
4 & 0.712 & 2.561 & 4.116 & 3.326 & 0.2773 & 0.0111 \\
5 & 0.747 & 2.663 & 4.259 & 3.430 & 0.2620 & 0.0050 \\
6 & 0.776 & 2.716 & 4.309 & 3.488 & 0.2552 & 0.0023 \\
7 & 0.802 & 2.746 & 4.318 & 3.543 & 0.2522 & 0.0008 \\
8 & 0.817 & 2.764 & 4.322 & 3.540 & 0.2504 & 0.0001 \\
9 & 0.848 & 2.775 & 4.285 & 3.562 & 0.2510 & 0.0008 \\
10 & 0.866 & 2.790 & 4.274 & 3.575 & 0.2489 & -0.0016 \\
11 & 0.876 & 2.799 & 4.273 & 3.586 & 0.2480 & -0.0022 \\
12 & 0.885 & 2.807 & 4.270 & 3.595 & 0.2473 & -0.0026 \\
13 & 0.893 & 2.813 & 4.266 & 3.602 & 0.2469 & -0.0030 \\
14 & 0.900 & 2.819 & 4.262 & 3.608 & 0.2465 & -0.0033 \\
& & & & & & \\
$\infty$ & 1 & 2.887 & 4.188 & 3.696 & 0.2426 & -0.0072 \\
\hline
\end{tabular}

sensitive than energies. The good agreement reflects the localization of the quantum wavefunctions in the vicinity of the periodic orbit.

At first sight it seems that the quantal expectation values for the mass polarization term reflect the classical behaviour much worse than all other quantities. However, this is not true. The occurrence of the discrepancy is related to the extremely small classical value of this quantity (recall that the outer electron is nearly frozen, hence $p_{1} \approx 0$ ). Asymptotically, the quantal values $\langle A\rangle$ all approach their classical limit in the same way, namely $\langle A\rangle \rightarrow\langle A\rangle_{c \mid}+\alpha_{A} / n+\mathcal{O}\left(1 / n^{2}\right)$. This is illustrated in figure 10 , where we plot the quantal expectation values $\left\langle p_{1} \cdot p_{2}\right\rangle / E$ against $1 / n$.

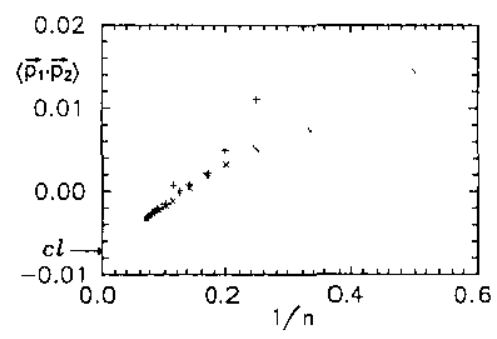

Figure 10. Scaled expectation values of the mass polarization term $\left\langle p_{1} \cdot p_{2}\right\rangle / E$. Both parity classes, ${ }^{1} S^{e}(+)$ and ${ }^{3} S^{e}(x)$, are shown. The classical limit is marked with an arrow.

More accurate complex quantal expectation values for the mass polarization term $\left\langle\boldsymbol{p}_{1} \cdot \boldsymbol{p}_{2}\right\rangle$ are tabulated for both symmetries in table 3 . These values can be used to correct the calculated energies and widths of the frozen-planet states for the effect of the finite nuclear mass $M$ in first-order perturbation theory $(1 / M=1.370933 \times$ $10^{-4}$ for the $\alpha$-particle),

$$
E_{M}^{(1)}=E+\frac{1}{M}\left(\left\langle p_{1} \cdot p_{2}\right\rangle-E\right)
$$


with the real and imaginary part of the complex energies $E$ listed in tables 1 and 5 . For example, for $n=2$ the perturbative results gives $E_{M}^{(1)}=-0.257333467-$ i0.000010537 compared to the exact value of $E_{M}=-0.257333473$ i0.000010537.

Table 3. Expectation values (in $10^{-3}$ au) for the mass polatization term $\left\langle p_{1} \cdot p_{2}\right\rangle$ of the $(n, 0,0)$ frozen planet states.

\begin{tabular}{rrr}
\hline$n$ & \multicolumn{1}{l}{${ }^{1} \mathrm{~S}^{e}$} & \multicolumn{1}{l}{${ }^{3} \mathrm{~S}^{\mathrm{e}}$} \\
\hline 2 & $20.85033+\mathrm{i} 0.18523$ & $3.59411+\mathrm{i} 0.02361$ \\
3 & $6.23728+\mathrm{i} 0.18379$ & $1.02370+\mathrm{i} 0.03245$ \\
4 & $0.99209+\mathrm{i} 0.04489$ & $0.46030+\mathrm{i} 0.00135$ \\
5 & $0.31080+\mathrm{i} 0.00280$ & $0.19874+\mathrm{i} 0.00001$ \\
6 & $0.10317+\mathrm{i} 0.00024$ & $0.07745+\mathrm{i} 0.01487$ \\
7 & $0.02869-\mathrm{i} 0.00293$ & $0.01315-\mathrm{i} 0.00102$ \\
8 & $0.00136-\mathrm{i} 0.04572$ & $-0.01308+\mathrm{i} 0.00009$ \\
9 & $0.01667+\mathrm{i} 0.03972$ & $-0.02615+\mathrm{i} 0.00025$ \\
10 & $-0.02880+\mathrm{i} 0.00105$ & $-0.03220+\mathrm{i} 0.00027$ \\
11 & $-0.03367-\mathrm{i} 0.00008$ & $-0.03443+\mathrm{i} 0.00020$ \\
12 & $-0.03471-\mathrm{i} 0.00043$ & $-0.03455+\mathrm{i} 0.00012$ \\
13 & $-0.03383+\mathrm{i} 0.00025$ & $-0.03356+\mathrm{i} 0.00007$ \\
14 & $-0.03201+\mathrm{i} 0.00010$ & $-0.03205+\mathrm{i} 0.00003$ \\
\hline
\end{tabular}

\subsection{The satellite series $(n, 0, l)$}

The semiclassical triple Rydberg formula (4) suggests the existence of quantum states with nodal excitations transverse to the periodic orbit (labelled by $l$ ), which preserves the collinear character of the motion. Such configurations are shown in figure 9 for $l=1(b)$ and $(e)$ and $l=2(c)$ and $(f)$. In the adiabatic Mo description such excitations in $r_{1}=R$ represent vibrational levels of the outer electron. It follows that the quantum numbers $l$ in the semiclassical and $\ell$ in the molecular classification scheme are identical. Figures $9(b)$ and $(c)$ shows that the vibrational wavefunctions in $r_{1}$ are not symmetric around the potential minimum. This anharmonicity is related to the asymmetric shape of the secondary potential well (see figure 4) in the Mo description.

If the particle motions are independent of each other the wavefunction separates in independent particle coordinates $r_{1}, r_{2}$. At a first glance the rectangular nodal pattern in figure 9 indicates a separation of the wavefunctions in these coordinates. Nevertheless, the radial (as well as the angular) motion of both electrons is highly correlated. It is the interaction with the inner electron which localizes the 'frozen' outer electron, and it is the dynamics of the inner electron which stabilizes the outer one (a static point-charge distribution generally results in unstable saddle configurations (Purcell 1976)). Thus figure 9 shows that even if the motion is highly correlated the wavefunction may (approximately) separate in single-particle coordinates. To argue about correlation properties from nodal patterns of wavefunctions alone may yield false conclusions.

In table 4 we list results for the lowest ${ }^{1} S^{e}$ satellite states for different values of $n\left(=n_{\mu}\right)$. The number of transverse nodes $l$ is limited for each $n$ (see section 2). For $n=3$ there exists only one satellite resonance $(l=1)$. The adiabatic approach presented here underestimates the possibility for formation of vibronic levels. The 
Table 4. Energies $E$, widths $\Gamma / 2$ and some expectation values for states of the satellite series $(n, 0, l)$ obtained by full quantum calculations. Only the real part of the expectation values are listed.

\begin{tabular}{|c|c|c|c|c|c|}
\hline$n, k, l$ & $-E$ & $\Gamma / 2$ & $\langle\cos \theta\rangle$ & $\langle\tan \alpha\rangle$ & $\left\langle r_{12}\right\rangle E$ \\
\hline $3,0,0$ & 0.14106416 & 0.00001174 & 0.534 & 2.215 & 3.73 \\
\hline $3,0,1$ & 0.13708822 & 0.00000248 & 0.552 & 3.126 & 5.67 \\
\hline $4,0,0$ & 0.08957080 & 0.00000202 & 0.712 & 2.561 & 4.12 \\
\hline $4,0,1$ & 0.08755962 & 0.00000660 & 0.521 & 3.256 & 6.01 \\
\hline $4,0,2$ & 0.08609767 & 0.00000079 & 0.609 & 4.139 & 7.68 \\
\hline $5,0,0$ & 0.06205356 & 0.00000056 & 0.747 & 2.663 & 4.26 \\
\hline $5,0,1$ & 0.06084021 & 0.00000098 & 0.737 & 3.314 & 5.70 \\
\hline $5,0,2$ & 0.05993822 & 0.00000145 & 0.743 & 4.053 & 7.26 \\
\hline $6,0,0$ & 0.04553867 & 0.00000020 & 0.776 & 2.720 & 4.31 \\
\hline $6,0,1$ & 0.04475856 & 0.00000036 & 0.763 & 3.293 & 5.60 \\
\hline $6,0,2$ & 0.04416129 & 0.00000650 & 0.747 & 3.907 & 6.95 \\
\hline $7,0,0$ & 0.03484264 & 0.00000037 & 0.802 & 2746 & 4.32 \\
\hline $7,0,1$ & 0.03431242 & 0.00000061 & 0.781 & 3.263 & 5.50 \\
\hline $7,0,2$ & 0.03389017 & 0.00000070 & 0.755 & 3.837 & 6.78 \\
\hline $8,0,0$ & 0.02751760 & 0.00000118 & 0.817 & 2764 & 4.32 \\
\hline $8,0,1$ & 0.02714168 & 0.00000094 & 0.795 & 3.223 & 5.38 \\
\hline $8,0,2$ & 0.02683582 & 0.00000101 & 0.781 & 3.729 & 6.49 \\
\hline
\end{tabular}

first vibronic state with $l=1$, which is bounded in the outer adiabatic potential well (see figure 4) appears for $n=n_{\mu}=6$.

Since the total error for the adiabatic energies is of the order of the $l$ oscillator spacing (see table 1), a comparison of absolute energies is not appropriate. However, a comparison of the energy differences of adjacent levels $l=0,1,2,3$ for $n=8$ yields (in units of $10^{-3}$ au) $\Delta E=0.376,0.306,0.253$ for the quantum calculation compared to $\Delta E=0.383,0.311,0.256$ for the adiabatic and $\Delta E=0.409,0.400,0.391$ for the semiclassical calculations. Thus the adiabatic treatment yields the correct internal structure of the $l$ multiplets. In particular it shows, that the level spacings diminish with increasing $l$, which follows from the asymptotic Coulomb-type behaviour of the adiabatic potential curves.

The expectation values for the satellite states listed in table 4 generally reflect the behaviour expected from the quasi-classical and from the adiabatic mo approach. $\langle\cos \Theta\rangle$ depends only weakly on the collinear radial vibration $l$ of the outer electron. Note the rather dramatic increase for $\langle\tan \alpha\rangle$ with $l$, which stems from the anharmonicity of the flat effective potentials for the outer electron. For the same reason the inter-electron distance $\left\langle r_{12}\right\rangle$ increases rapidly with $l$. The decay widths tend to become larger, which is consistent with the mo and with the quasi-classical approach.

\subsection{The satellite series $(n, k, 0)$}

In the preceding section we investigated the (radial) vibrational mode of the outer electron described by the quantum number $l$, i.e. excitations within the collinear arrangement of the electrons. We now focus on excitations of the bending degree 
of freedom $\Theta$ described by the semiclassical quantum number $k$ or, equivalently, on excitations in the Mo coordinate $\lambda$, equation (14), with Mo quantum number $n_{\lambda}$. In the adiabatic treatment the dynamics decouples into motion of the outer electron (vibrational $R$ motion) and of the inner electron, which for fixed $R$ separates in prolate spheroidal coordinates $\lambda, \mu$. To test these predictions we plot in figure 11 the probability densities of the $a b$ initio three-dimensional quantum wavefunctions for fixed distances $R$ of the outer electron. The figure then depicts the conditional probability for finding the inner electron in the coordinate space relative to the axis $R$ connecting the nucleus and the outer electron. We choose $R$ as the classical expectation value $\left\langle r_{1}\right\rangle_{\mathrm{cl}}$ for the radial distance of the outer electron along the classical periodic orbit of figure $1(a)$.
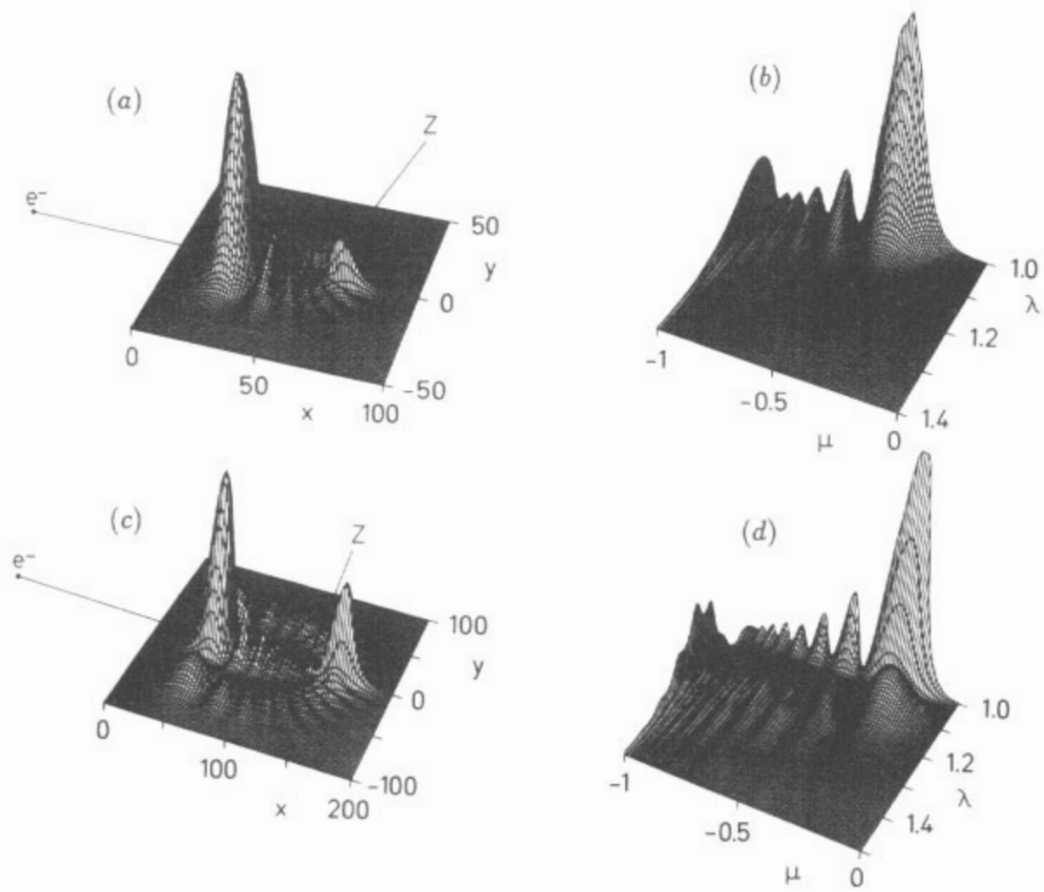

Figure 11. Conditional probability densities for the inner electron with respect to the fixed axis $\boldsymbol{R}$ between the nucleus and the outer electron. The $(n, k, l)=(6,0,0)$ state is shown in Euclidian coordinates of the inner electron in part $(a)$ and in spheroidal coordinates in $(b)$. Parts $(c)$ and $(d)$ show the $(9,1,0)$ state. The position of the nucleus $(Z)$ is indicated in parts $(a)$ and $(c)$.

Part (a) of figure 11 indeed shows that the inner electron wavefunction is of molecule-type character with maximal polarization along the axis between nucleus and outer electron. The state exhibits no off-radial excitations and is described by $n_{\mu}=6, n_{\lambda}=0$ in the Mo classification scheme. Part $(c)$ shows a state with $n_{\mu}=9$ and one nodal off-axis excitation $n_{\lambda}=1$. The adiabatic picture not only describes the number of nodes in the wavefunctions correctly, but also predicts the shape of the nodal surfaces: they are of hyperbolic and elliptical character with the nucleus as one focus. This nodal structure becomes more obvious in parts $(b),(d)$ of figure 11, where we represent the corresponding wavefunctions in prolate spheroidal 
coordinates $\lambda, \mu$. The nodal surfaces appear as nearly straight lines, which proves the approximate separability of the $a b$ initio quantum wavefunctions in these coordinates.

The validity of the adiabatic treatment is further established by the observation that the structure of the wavefunctions in figure 11 is insensitive to variations in $R$, as long as $R$ is chosen such that we can speak of an outer and of an inner electron. The adiabatic approach loses its applicability for smaller values of $R$, where the outer electron has penetrated the potential barrier, but the probability densities of the corresponding exact wavefunctions are negligible there. For $R \rightarrow \infty$ the structure of the wavefunctions remains, but the absolute probability densities decrease if $R$ passes the classical localization point $\left\langle r_{1}\right\rangle_{\mathrm{cl}}$.

In terms of the semiclassical classification scheme the nodal excitations $n_{\lambda}$ are identified with bending nodes (labelled by $k$ ) transverse to the periodic orbit. For a semiclassical explanation of the elliptical and hyperbolic topology of the nodal lines an investigation of the curvature of the classical torus structure is necessary, but this is beyond the simplest semiclassical approach presented here.

Finally, we focus on the correspondence between the classical and the quantum dynamics by showing in figure 12 a combined plot of the inner and outer electronic densities, which images the charge distribution of the entire two-electron atom. A global space filling charge distribution is obtained by an overall rotation around the centre of mass (nucleus). The probability density of the outer electron of the state $(6,0,0)$ is obtained in analogy to figure 11 by drawing a cut through the full wavefunction at a fixed radial distance $r_{2}$ of the inner electron. The wavefunction of the outer electron (left-hand side of figure 12) appears as the bump far away from the nucleus $Z$. It just resembles the ground-state oscillator-like wavefunction $(l=0)$ in the outer well of the Bo potential and turns out to be extremely nonhydrogenic. The inner wavefunction is taken from figure 11(a). A comparison with the quasiperiodic classical motion shown in figure $12(b)$ illustrates the classical-quantal correspondence of the electron-pair motion. The radial charge distribution shown somewhat resemble that of an (uncorrelated) state with an outer electron having maximal angular momentum. In such a state the trapping of the outer electron would occur through the centrifugal barrier of the very high angular momentum state. Here, however, the trapping is caused by the electron correlation.

Summarizing the results for the principal and satellite series, we have established a classification for the frozen-planet configurations in terms of the semiclassical quantum numbers $(n, k, l)$ associated with local coordinates of the periodic orbit and the Mo set $\left(n_{\mu}, n_{\lambda}, \ell\right)$ associated with molecular-type coordinates, which (locally) are identical. The exact wavefunctions of the problem show the quasi-separable behaviour in these coordinates. Energies calculated within the two approximate approaches reproduce the exact quantum results quite accurately.

\section{Decay of the resonant states}

There are, in principle, two mechanisms which lead to the decay of the planetary configurations described in the preceding sections: radiative and non-radiative decay. We first discuss the non-radiative particle decay, i.e. autoionization of the resonances.

The classical decay properties are essentially described by the Poincaré surface of section shown in figure 2. A trajectory confined to one of the closed tori never ionizes. Ionization of collinear classical configurations only takes place for trajectories 

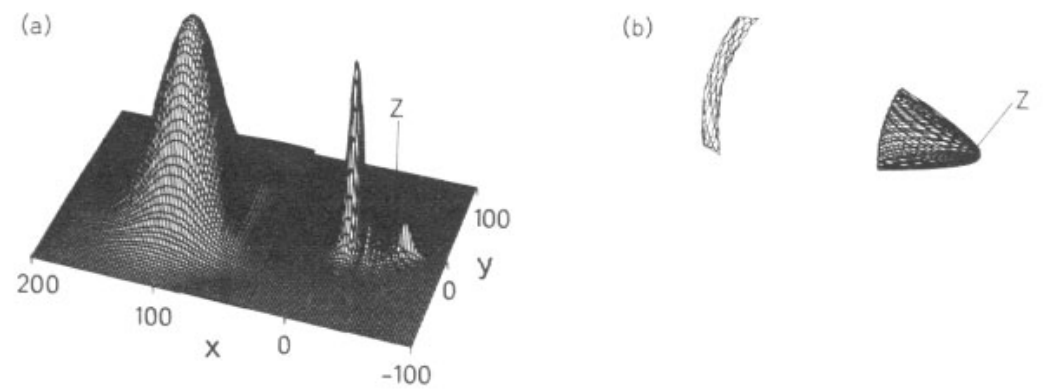

\begin{abstract}
Figure 12. Image of the charge distribution of the helium atom in the $(6,0,0)$ state, part (a). The right part is the probability distribution of the inner electron at fixed radial distance $R$ of the outer electron; the left part is the probability for the outer electron at fixed inner electron radius $r_{2}$. The fixed values were chosen as the classical outer turning points of the electrons. Part (b) shows a typical classical trajectory of the two electrons. The trajectory is confined to a torus close to the frozen-planet periodic orbit in phase space. The position of the nucleus $(Z)$ is indicated in part (a).
\end{abstract}

starting on one of the non-closed phase space manifolds, for which the outer electron (regularly) escapes to infinity. For non-collinear configurations the situation is similar: as long as the trajectories are confined to tori the motion is quasi-periodic and bounded. However, the phase space structure beyond the stability island becomes more complicated and eventually allows for chaotic ionization (for a precise definition of chaotic ionization see, for example, Eckhardt (1987) or Smilansky (1992)). In principle, autoionization can also occur for classical configurations inside the stability island due to Arnold diffusion (Lichtenberg and Lieberman 1983), but we did not observe this process even for time scales of some thousand periods.

Semiclassically, we can only estimate the decay times. The classical trapping of the electrons around the frozen-planet configuration is not caused by barriers in the electron-pair potential but has a pure dynamical origin. However, in analogy to the (semiclassical) penetration through a 'static' potential barrier there exists 'dynamical' tunnelling (Davis and Heller 1981, Bohigas et al 1990) between different phase space regions. Thus a two-electron state of the type $(n, k, l)$, which is localized on a torus near the periodic orbit, may decay semiclassically by tunnelling through the tori of bounded motion into the region of manifolds outside the island representing ionizing motion. The probability $P(E)$ for such a decay process is given by

$$
P(E) \sim \exp \left(-\alpha S_{\mathrm{dt}} / \hbar\right)
$$

where $S_{\mathrm{dt}}$ is some action characterizing the dynamical tunnelling process analogous to the barrier integral performed for the tunnelling trajectory in a barrier tunnelling process. The scaling property (2) for Coulomb systems yields $S_{\mathrm{dt}}=S_{\mathrm{dt}}^{\mathrm{sc}} / \sqrt{-E}$, where the scaled action $S_{\mathrm{dt}}^{\mathrm{sc}}$ is now energy independent. Thus the semiclassical widths of the resonances tend to decrease exponentially with increasing excitation. Such a behaviour is also consistent with the semiclassical theory of EBK wavefunctions, which are exponentially localized in the neighbourhood of the quantized tori (Berry 1989).

In table 5 we summarize the widths of the ${ }^{1} S^{e}$ and ${ }^{3} S^{e}$ resonances of the principal series. They were calculated fully quantum mechanically using the complex rotation method described in section 4. Highly accurate calculations in large basis sets are necessary to pin down the precise values of the extremely small imaginary parts of 
the complex energy eigenvalues. The widths $\Gamma / 2$ indeed decrease exponentially with increasing nodal excitation $n \sim 1 / \sqrt{-E}, \ln \Gamma / 2 \simeq-0.71 n$, although they fluctuate strongly around the average trend. Thus the resonances appear as bound states in the continuum in the limit of large excitation even though the number of open channels increases tremendously with increasing energy (more than 100 channels are open for the $n=14$ state!). The number of open (autoionization-) channels into which the states can decay are listed in column 5 of table 5 .

Table 5. Total decay widths $\Gamma / 2$ (in $10^{-3}$ au) of the singlet and triplet states of the principal series $(n, 0,0)$. Column 4 gives the number $K$ of open channels into which the states can decay.

\begin{tabular}{rllr}
\hline$n$ & $\Gamma / 2\left({ }^{1} S^{e}\right)$ & $\Gamma / 2\left({ }^{3} S^{e}\right)$ & \multicolumn{1}{r}{$K$} \\
\hline 2 & 0.010564 & 0.006789 & 3 \\
3 & 0.011739 & 0.004409 & 6 \\
4 & 0.002024 & 0.000179 & 10 \\
5 & 0.000560 & 0.000033 & 15 \\
6 & 0.000202 & 0.000376 & 21 \\
7 & 0.000368 & 0.000143 & 28 \\
8 & 0.001184 & 0.000022 & 36 \\
9 & 0.000525 & 0.000035 & 45 \\
10 & 0.000058 & 0.000030 & 55 \\
11 & 0.000023 & 0.000019 & 66 \\
12 & 0.000022 & 0.000010 & 78 \\
13 & 0.000014 & 0.000005 & 91 \\
14 & 0.000004 & 0.000002 & 105 \\
\hline
\end{tabular}

The parity splittings $\Delta E=E\left({ }^{1} \mathrm{~S}^{\mathrm{e}}\right)-E\left({ }^{3} \mathrm{~S}^{\mathrm{e}}\right)$ between doublet states of different exchange symmetry also decrease exponentially $(\ln |\Delta E| \approx-1.4 n)$, as has already been discussed in connection with figure 8 . The semiclassical explanation for this is analogous to the semiclassical treatment of the decay widths. Only the exponentially small overlap of the wavefunctions localized on the different $\left(r_{1} \leftrightarrow r_{2}\right)$ symmetry images of the quantized tori contribute to the exchange integral, which determines the parity splitting. The dynamical tunnelling process now occurs twice, once for the penetration to the outside region of the stability island and once for the penetration inside the symmetry image of the island. This explains why the exponent for the parity splitting is approximately twice the exponent for the decay widths.

In the adiabatic description of section 3 two different processes lead to nonradiative decay. Assume for example, that the outer electron is in the ground state of the outer potential well in figure 3. Then the resonance can decay by

(i) barrier penetration of the outer electron into the inner region and successive energy transfer between the electrons leading to escape; and

(ii) transitions due to non-adiabatic couplings, where the inner electron decays to a less excited state and transfers the excess energy to the outer electron, or where the inner electron changes to a less polarized state, which no longer supports a minimum for the outer electron.

In the first case tunnelling probabilities are obtained by calculating the barrier penetration integral of the Bo potential curves. Hence these decay probabilities decrease exponentially, but stronger $(\ln \Gamma \simeq-1.1 n)$ than for the quantal results $(\ln \Gamma \approx-0.71 n)$. Such differences are not surprising, since transitions of the second 
type are neglected in the adiabatic case. In addition, the adiabatic treatment becomes somewhat obscure near the inner part of the barrier region, where the underlying assumption of a slowly moving outer electron breaks down.

Decay of the second type occurs by non-adiabatic transitions of the inner electron between different BO potential curves. As pointed out at the end of section 3 the non-adiabatic couplings are small near the minimum of the outer potential well (Thürwächter 1992), but they may get important near the top of the barrier, where avoided crossings between the upper potential curve of an $N$ manifold with the second highest curve of the $(N+1)$ manifold exist (see figure 3 ). Non-adiabatic transitions in the region of avoided crossings are associated with branch points in the complex $R$ plane connecting the potential curves (Groszdanov and Solovev 1990). In a WKB approach the transition probability is then given by a contour integral in the complex $R$ plane including the branch point (Landau and Lifshitz 1965). Such an analysis shows that the most important couplings are those between an upper BO potential $\left(N, n_{\lambda}, n_{\mu}\right)=(N, 0, N-1)$ and the potential curve $(N+1,1, N-1)$ of the next higher manifold. Thus in the adiabatic description those transitions are favoured, for which the number of off-radial nodes $n_{\lambda}$ changes by +1 , keeping the radial nodes $n_{\mu}$ constant.

Finally, we compare the total particle decay rates with those induced by radiative transitions. Since an accurate calculation of the total radiative decay is too elaborate we will only give estimates. The lifetime of the entire two-electron resonance is largely limited by the lifetime of the inner electronic Stark-like state. Neglecting the influence of the outer electron we can directly transfer the calculations for hydrogenic Stark states (Hiskes et al 1964) to the $\mathrm{He}^{+}$system by scaling the results with $Z^{-4}$. The average radiative transition probability for a hydrogenic level $N$ is proportional (Herrick 1975) to $\ln N / N^{5}$. Since the non-radiative decay rates decrease exponentially, there exists a critical value $N_{\mathrm{cr}}$, which divides the region of non-radiative-dominated decay from the region of radiative-dominated decay. Extrapolation of the results for the decay widths (table 5 ) yields $N_{\mathrm{cr}} \approx 20$, which corresponds to a lifetime of approximately $0.5 \times 10^{-6} \mathrm{~s}$. For states with $N>N_{\mathrm{cr}}$ the resonances decay mainly by radiative transitions.

\section{Dependence on nuclear charge $Z$}

The classical frozen-planet configurations analysed in this paper exist for all twoelectron atoms or ions with nuclear charges $1<Z<\infty$. For the hydrogen negative ion $(Z=1)$ the potential $-Z / r_{1}+1 /\left(r_{1}-r_{2}\right)$ for the outer electron in the collinear arrangement is purely repulsive and the $\mathrm{H}^{-}$system does not support these types of configuration. For $Z=\infty$ (independent-electron approximation) the electronic motions are independent of each other, which excludes the formation of an outer potential well due to the electron-electron interaction. Thus the configurations discussed here do not exist in an independent electron description, which would be the natural starting point for a perturbative treatment in powers of $1 / Z$.

We consider first the classical properties of the frozen-planet configurations in order to study the dependence on the nuclear charge $Z$. As argued in section 2 the existence of the long-lived resonant states is related to the stability of the corresponding classical motion. We find that the fundamental collinear periodic orbit of the configuration (depicted in figure $1(a)$ for $\mathrm{He}$ ) is fully stable for nuclear charges 
$Z \leqslant 12.786$. At least for sufficient high excitations this implies the existence of longlived resonances for the isoelectronic sequence with $1<Z \leqslant 12$. The investigation of the $Z$-dependence of the Poincaré surfaces of section analogous to that of figure 2 shows, that tori are more and more destroyed and break up into chains of stable and unstable fixed points with increasing $Z$. However, the overall phase space structure turns out to be robust with respect to variations in $Z$ and the integrable structure near the fundamental fixed point survives for $Z \leqslant 12$.

The classical winding numbers describing the evolution of nearby trajectories are listed in table 6 together with the scaled actions of the periodic orbit. These entries are the classical ingredients for the calculation of semiclassical energies with the triple Rydberg formula (4).

Table 6. Dependence on the nuclear charge $Z$ of the scaled radial distances $r_{i}^{\text {sc }}$ of the outer and inner turning points of the outer $(i=1)$ and inner (2) electron for the frozen-planet periodic orbit. The scaled action $S^{3 c}$ and both winding numbers $\gamma_{i}$ are given in columns 4,5 and 6 .

\begin{tabular}{lcllll}
\hline$Z$ & $r_{1}^{\mathrm{sc}}$ & $r_{2}^{\mathrm{sc}}$ & $S^{\mathrm{sc}}$ & $\gamma_{1}$ & $\gamma_{2}$ \\
\hline 1.01 & $152.76-152.76$ & $0-1.01$ & 0.7142 & 0.5000 & 0.0000 \\
1.1 & $17.90-17.90$ & $0-1.10$ & 0.7789 & 0.4992 & 0.0017 \\
1.5 & $6.51-6.51$ & $0-1.55$ & 1.0839 & 0.4859 & 0.0268 \\
2 & $5.78-5.80$ & $0-2.15$ & 1.4915 & 0.4616 & 0.0677 \\
3 & $6.60-6.70$ & $0-3.47$ & 2.3553 & 0.4116 & 0.1366 \\
4 & $7.94-8.16$ & $0-4.87$ & 3.2550 & 0.3666 & 0.1861 \\
5 & $9.42-9.78$ & $0-6.32$ & 4.1754 & 0.3266 & 0.2225 \\
10 & $17.32-18.67$ & $0-13.93$ & 8.9202 & 0.1598 & 0.3204 \\
\hline
\end{tabular}

Columns 2 and 3 of table 6 contain the scaled distances $r_{i}^{\text {sc }}$ for the turning points of the electrons along the periodic orbits. The shapes of the frozen-planet orbits depend somewhat strangely on the nuclear charge. The inner electron radial extent $r_{2, \max }^{\mathrm{sc}}$ decreases monotonically with decreasing $Z$, but not proportional to $Z$. The outer turning point of the outer electron $r_{1, \max }^{\mathrm{sc}}$ is minimal for helium. For $Z>2$ the outer electron distance $r_{1, \max }^{s c}$ increases monotonically and can be approximated by $r_{1, \max }^{\mathrm{sc}} \stackrel{Z \rightarrow \infty}{\longrightarrow} 2 Z$. For $Z<2$, however, $r_{1, \max }^{\mathrm{sc}}$ again increases as $Z$ approaches one and behaves asymptotically as $r_{1, \max }^{\mathrm{sc}} \stackrel{Z \rightarrow 1}{\longrightarrow} 3 / 2(Z-1)$. This should be compared with results for independent electron motion, where radial distances generally scale proportional to $Z$. For small nuclear charges $Z$ the outer electron is spatially well separated from the inner electron. With increasing $Z$, however, the turning points of the electrons approach each other more and more closely.

We now discuss the dependence of the adiabatic potentials on the inner electronic Mo quantum numbers. The shapes of the potentials are similar to those for helium $(Z=2)$. The minimal principal quantum number $N_{\min }$, for which the Bo potentials $U_{0, N-1}$ show an outer minimum increases with $Z$. For $Z=2,3$ and 4 the values of $N_{\min }$ are 5,11 and 21, respectively: with increasing nuclear charge the polarization of the inner electron wavefunction must be larger to overcome the stronger nuclear attraction for the outer electron. On the other hand, for $Z=1.23$ the mo state $N=2$ with $\left(n_{\lambda}=0, n_{\mu}=1\right)$ already supports stable vibrational motion. For a convenient comparison of the BO potentials for different $Z, n_{\lambda}, n_{\mu}$ we derive and apply an approximate scaling property for the potential curves. The asymptotic ex- 

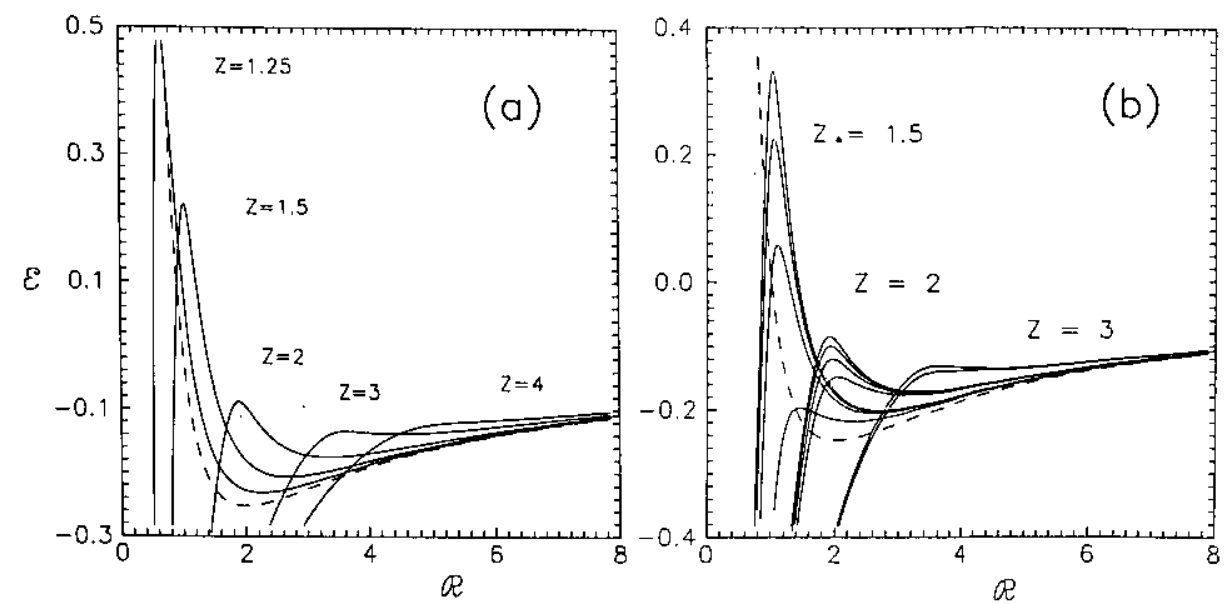

Figure 13. Scaled $n_{\lambda}=0$-potential surfaces are shown in $(a)$ for various nuclear charges $Z$. The broken curve represents the universal dipole potential. $(b)$ is similar to $(a)$, but in addition different $n_{\mu}$ potential curves are shown for several charges $Z$.

pansion in $1 / R$ of Mo energies for the two-centre problem with charges $Z$ and -1 and $m=0$ reads (Komarov and Slavyanov 1968):

$E\left(n_{\lambda}, n_{\mu}, Z ; R\right)=-\frac{Z}{2 N^{2}}+\frac{1-Z}{R}+\frac{3 N\left(n_{\mu}-n_{\lambda}\right)}{2 Z R^{2}}+\mathrm{O}\left(R^{-3}\right)$.

Omitting terms of order $1 / R^{3}$ and introducing the new length and energy measures

$$
\mathcal{R}=\frac{2 Z(Z-1)}{3 N\left(n_{\mu}-n_{\lambda}\right)} R \quad \mathcal{E}=\frac{3 N\left(n_{\mu}-n_{\lambda}\right)}{2 Z(Z-1)^{2}}\left(E+\frac{Z^{2}}{2 N^{2}}\right)
$$

yields a universal form for the dipole potential (33):

$$
\mathcal{E}=-\frac{1}{\mathcal{R}}+\frac{1}{\mathcal{R}^{2}} \text {. }
$$

The universal dipole potential is shown as a broken curve in figure 13. Expressing the Bo energies and distances in terms of $\mathcal{E}$ and $\mathcal{R}$ allows for a compact representation of potentials surfaces belonging to different values of $Z, n_{\lambda}$ and $n_{\mu}$ as depicted in figure 13. Part (a) shows one typical member of each potential series for different nuclear charges. Whereas the energy curve obtained within the dipole approximation is independent of $Z, n_{\lambda}$ and $n_{\mu}$, it is only in the large $\mathcal{R}$ limit that the Bo potentials coincide with the dipole potential. In addition to the strong dependence on the nuclear charge $Z$, the Bo potentials show large deviations from the universal dipole form, which indicates the inadequacy of the dipole approximation to describe the well structure and the frozen-planet configurations. For helium the dipole curve exhibits a well where the exact potentials have maxima. The inclusion of higher terms in the multipole expansion (33) leads to better agreement with the full BO curves in the asymptotic regime and for distances $R$ close to the outer minimum. However, the potential maxima of the BO curves cannot be reproduced by adding further multipole terms. The barrier structure of the во curves results from a rearrangement of the wavefunction for the inner electron at small values of $R$ and cannot be described by the asymptotic multipole expansion. 
Finally, figure $13(b)$ shows the formation of vibronic levels for varying $N\left(n_{\lambda}=0\right)$ and $Z=1.5,2,3$. For smaller $Z$ the number of vibrational levels grows drastically. The helium atom turns out to possess the optimal charge within the isoelectronic sequence for the formation of the frozen-planet configurations, as it gives rise to the most pronounced potential wells in the adiabatic description.

\section{Relation to previous theoretical and experimental work}

Molecular coordinates have also been used for the description of intra-shell resonances of symmetrically excited electron states (Feagin and Briggs 1988, Rost and Briggs 1991). For these states the inter-electron distance $r_{12}=r_{1}-r_{2}$ is used as an adiabatic coordinate. Though the states considered here are of an entirely different type, the results are in some sense related: both approaches lead to an adiabatic behaviour of a part of the combined electron motion and to the separability of the non-adiabatic part in molecular coordinates for fixed values of the adiabatic parameter (Rost et al 1991). While variation in $r_{12}$ leads to a variation in the MO nodal structure of the symmetrically excited states and nodal lines might vanish or new nodes might appear (Rost et al 1991), the $\lambda, \mu$-structure of the asymmetrically excited planetary states discussed here is insensitive to variations in $R=r_{1}>r_{2}$.

We can extract two extreme classes of states from the multitude of doubly excited resonances: states of symmetric excitation, which are localized along the energetically preferred equilibrium position $\Theta=\pi$, and asymmetric resonances with both electrons located near $\Theta=0$. Although the two classes behave differently, they are subject to similar symmetry principles. Both types of resonances are located near collinear symmetry planes and in both cases the bending motion proves to be stable (Richter and Wintgen 1990a, Ezra et al 1991) and can be separated to a good approximation.

The connections between intra-shell multiplets and the particular inter-shell configurations discussed here are not yet fully developed. There are some indications that the energies of the frozen-planet states can be diabatically connected to the highest energy levels of each intra-shell manifold in the limit $Z \rightarrow \infty$ if $Z$ is considered as a continuous parameter. A more detailed investigation of the $Z$-dependence of the resonances is necessary to establish such a connection. Even if an energy-correlation diagram would support such a point of view, we emphasize that the structure of the corresponding intra-shell states is completely different and changes strongly with varying $Z$.

The most common labelling scheme for doubly excited states, the $N_{1}(K, T) N_{2}$ notation, was introduced by Herrick and co-workers (Herrick and Sinanoglu 1975, Herrick 1983). Starting from a group theoretical approach, they derived two approximate quantum numbers $K$ and $T$ to characterize 'doubly excited symmetry basis' (DESB) functions. The DESB functions approximately diagonalize the electronelectron interaction within the degenerate hydrogenic manifolds of fixed principal quantum numbers $N_{1}$ and $N_{2}$ of the outer and inner electron respectively. Lin (1984) re-interpreted Herrick's classification by labelling hyperspherical curves calculated with an adiabatic ansatz for the hyperspherical radius $\rho=\sqrt{r_{1}^{2}+r_{2}^{2}}$ (Macek 1968, Sadeghpour and Greene 1990). A derivation of Herrick's quantum numbers within the molecular framework is reviewed in Rost and Briggs (1991). The quantum number $T$ describes the projection of the total angular momentum $L$ in the body-fixed frame with the $z$-axis directed along the inter-electron vector $\boldsymbol{R}$. For 
$S$ states as discussed here, $T$ is always zero. The Stark-type quantum number $K$ describes the angular correlation of the wavefunctions in the limit $N_{1} \gg N_{2}$, ie. $K \approx-N_{2}\langle\cos \Theta\rangle$. Within this labelling, the frozen-planet states should carry the label $K=2 n_{\lambda}-\left(N_{2}-1\right)$. However, we emphasize that $K$ only can serve as a label, whereas $n_{\lambda}$ describes the nodal surfaces and reflects the approximate separability of the wavefunctions in the Mo coordinates. The principal quantum number $\mathrm{N}_{2}$ of the inner electron in Herrick/Lin's classification is directly related to the nodal quantum number $n$ of the frozen-planet states: $N_{2}=n+1$. However, $N_{1}$ can no longer be assigned a meaningful value: as was demonstrated in section 5 the vibrational motion of the outer electron is described by an extremely non-hydrogenic oscillator-like wavefunction. There is no similarity in the radial dependence of the DESB states and of the frozen-planet states discussed here.

There is also current experimental progress in the spectroscopy of planetary atoms (Camus et al 1989). Eichmann et ai (1990) prepared laser excited states of barium with principal quantum numbers $n_{1} \approx 90, n_{2} \approx 40-80$. They interpreted observed structures as Stark-induced states of the inner electron in the field of the slowly moving outer electron. Although their results suggest formation of the configurations discussed here, a direct connection of their observations with our calculations is not possible because the experimental resolution is not sufficiently high. However, there are now improved measurements (Eichmann $e t$ at 1992) which give some evidence that electron-pair configurations of the type discussed here were excited.

A formula similar to equation (4),

$$
E_{N}=-(Z-\sigma)^{2} /(N-\mu)^{2}
$$

has been used previously to parametrize particular intra-shell resonances (the socalled 'Wannier resonances') (Rau 1983, Molina 1989, Rost and Briggs 1989). The quantum defect $\mu$ and the screening charge $\sigma$ were introduced heuristically (Molina 1989). In contrast, the quantities entering the Rydberg formula (4) have a well defined origin in terms of classical properties and the general appearance of the formula is simply a direct result of the classical scaling property of the action (see equation (3)). Recently, it has been shown that such a classical correspondence is also valid for certain intra-shell resonances (Ezra et al 1991, Wintgen et al 1992). The semiclassical derivation of a double Rydberg-type formula for intra-shell states was also performed by Solov'ev (1985).

\section{Summary and conclusions}

In the present paper we have studied some properties of a certain class of resonant states of two-electron atoms and ions, namely the so-called frozen-planet configurations of highly doubly excited electrons. The problem has been investigated from several different points of view-classically, semiclassically and quantum mechanically (both exactly and in an adiabatic approach).

The analysis of the classical electron-pair motion and the associated phase space regions proves the stability and the near-integrability of the underlying classical dynamics. We emphasize that these are local properties of the systems in that they do not describe the global structure of the full phase space, which also supports chaotic motion (Ezra et al 1991). Classically, the configurations are stable only for 
$Z \lesssim 12$. Recalling also the extreme angular and radial correlation of the electrons, this demonstrates the highly non-perturbative nature of these configurations. It is hard to imagine how a perturbative treatment in $1 / Z$ could explain the existence and the structure of such states by starting with an independent-particle model.

The quantitative classical analysis is a necessary ingredient for the subsequent semiclassical treatment and also serves as motivation (and justification) for the adiabatic quantum mechanical treatment. A simplified semiclassical torus quantization of the phase space region around the central frozen-planet periodic orbit yields the $a b$ initio triple Rydberg series (4) which is exact in the semiclassical limit $n \rightarrow \infty$. The semiclassically derived quantum numbers $(n, k, l)$ have a simple interpretation in terms of nodal numbers along fundamental modes, i.e. along the periodic orbit and along the (decoupled) perpendicular degrees of freedom. The semiclassical treatment also accounts for expectation values and, at least semi-quantitatively, for the autoionization rates and doublet splittings.

The angular correlation between the electrons corresponds to a highly polarized inner electron localized along the axis connecting the outer electron with the nucleus. The inner electron wavefunction is then of molecular Mo type, which is a coherent superposition of all single-particle angular momenta $l_{i}$. The adiabatic approximation involving the MO ansatz for the inner electron yields potential curves for the radial coordinate of the outer electron which show pronounced minima for sufficiently high inner mo quantum numbers. The dynamical radial barrier of the outer electron is mimicked in the adiabatic description by the static barrier of the potential wells. Even though the calculated adiabatic energy levels cannot compete with the accuracy of the semiclassical results, the structure of the spectra and of the wavefunctions involved are adequately described within the adiabatic framework. The quantum numbers derived from the adiabatic approximation, $\left(n_{\lambda}, n_{\mu}, \ell\right)$ are in one-to-one correspondence with the semiclassically derived set $(n, k, l)$.

Using Sturmian basis functions and perimetric coordinates for the calculation of matrix elements we have transformed the Schrödinger equation of the three-body Coulomb problem into a sparse complex symmetric matrix eigenvalue problem in a fully quantum mechanical treatment. Diagonalization of the Hamiltonian matrix yields highly accurate results for the energies, expectation values, decay widths and wavefunctions of the resonant states. The results reported here represent by far the highest excited states and the most accurate $a b$ initio calculations performed so far for any three-body Coulomb complex. The calculations fully support the results and conclusions of the classical picture. In addition, we could prove the exponential stability of the resonant states against autoionization. The quantum mechanical solutions precisely reflect the dynamical localization of the frozen electron. More importantly, the nodal structure of the wavefunctions involved not only accurately reflects the approximate separability of the inner and the outer electrons, but also the spheroidal separation of the inner electron motion. This novel dynamical symmetry for two-electron atoms is fundamentally different from the symmetry considerations and classification schemes discussed so far in the literature. The approximate separability of the full two-electron wavefunctions is not necessarily obvious from the adiabatic treatment, which, in principle, allows for mixing of inner coordinates with the adiabatic coordinate, see, e.g., the discussion in Rost et al (1991) and Wintgen et al (1992).

The preparation and the observation of the resonances presumably requires nontrivial experimental effort. Because of the exponential localization of the outer elec- 
tron any transition matrix element involving the ground state nearly vanishes. Highly excited $\mathrm{He}^{*}$ atoms or $\mathrm{He}^{+*}$ ions) are necessary as intermediate states for an efficient photo-excitation or photo-recombination, respectively.

A more convenient experimental way might be the multi-step laser excitation of alkaline earth atoms such as strontium or barium (Camus et al 1989, Eichmann et al 1990, 1992). For these systems with two valence electrons, the effect of the residual core must be considered. Classically, this does not alter our conclusions significantly. Only the inner electron penetrates the core, which results in some short-ranged modification of the nuclear attraction. Quantum mechanically, however, the polarizability of the inner electron is of crucial importance for the dynamical localization of the outer electron and this is diminished due to the presence of non-vanishing quantum defects of the core-penetrating inner electron. However, the simple scaling arguments (2) indicate that the electron-electron interaction, which scales proportional to the energy $E$, will overcome, for sufficiently high excitation, the core effects which scale proportionally to $E^{3 / 2} \sim 1 / n^{3}$. Very recently, extremely long-lived states were detected in highly doubly excited strontium atoms (Eichmann et al 1992), but an unambiguous identification of the character of these states has not been possible to date.

An alternative approach would be to look for similar configurations in other threebody systems. It is immediately obvious from the classical analysis that the dynamically localized outer electron can be replaced by heavy negatively charged particles (such as kaons $\mathrm{K}^{-}$or antiprotons $\overline{\mathrm{p}}$ ) without changing inner electron dynamics essentially. Indeed, the present mechanism has been proposed as a trap for anti-particles (Richter et al 1991) and unexpectedly long-lived states have been found experimentally in such systems recently (Yamazaki et al 1989, Iwasaki et al 1991). In these experiments metastable states were populated by stopping negative hadrons in liquid helium. The precise mechanism for the formation of these long-lived states could not be extracted from the experimental data. However, the fact that such long-lived states were not observed in liquid nitrogen and other targets (Yamazaki 1992) suggests that the two (valence) electrons of the helium atom might be of importance for the existence of these states.

\section{Acknowledgments}

We would like to thank U Eichmann, J M Rost, W Sandner, R Thürwächter, and A Vollweiter for stimulating discussions. KR is grateful for the kind hospitality extended to him during a stay at the Leningrad State University (now St Petersburg State University). EAS is indebted to the Sonderforschungsbereich 276 for the hospitality at the University of Freiburg. This work was supported by the Deutsche Forschungsgemeinschaft under contract Wi877/2 and within the Sonderforschungsbereich 276 at the University of Freiburg.

\section{References}

Berry M V 1989 Proc. R. Soc. A 423219

Blümel R and Reinhardt W P 1991 Directions in Chaos vol 4, ed B L Hao et al (Hongkong: World Scientific)

Bohigas O, Tomsovic S and Ullmo D 1990 Phys. Rev. Lett. 641479 
Braun P A, Ostrovsky V N and Prudov N V 1990 Phys. Rev. A 426537

Camus P, Gallagher T F, Lecomte J M, Pillet P and Boulmer J 1989 Phys. Rev. Lett. 622365

Davies M J and Heller E J $1981 \mathrm{~J}$. Chem. Phys. 75246

Delande D, Bommier A and Gay J C 1991 Phys. Rev. Lett. 66141

Domcke M, Xue C, Puschmann A, Mandel T, Hudson E, Shirley D A, Kaindl G, Greene C H, Sadeghpour H R and Petersen H 1991 Phys. Rev. Lett. 661306

Eckhardt B 1987 J. Phys. A: Math Gen. 205971

Eckhardt B and Wintgen D 1991 J. Phys. A: Math. Gen. 244335

Eichmann U, Lange V and Sandner W 1990 Phys. Rev. Lett. 64274 1992 Phys. Rev. Leth 6821

Ezra G S, Richter K, Tanner G and Wintgen D 1991 J. Phys. B: At. Mol. Opt. Phys. 24 LA13

Feagin J M and Briggs J S 1986 Phys. Rev. Lett. 57984

1988 Phys. Rev. A 374599

Frost A A, Inokuti M and Lowe J P 1964 J. Chem. Phys. 41482

Gailitis M and Damburg R 1963 Proc. R Soc. 82192

Groszdanov T P and Solov'ev E A 1990 Phys. Rev. A 422703

Gutzwiller M C 1990 Chaos in Classical and Quantum Mechanics (New York: Springer)

Harris P G, Bryant H C, Molıagheghi A H, Reeder R A, Tang C Y, Donahue J B and Quick C R 1990 Phys. Rev. A 426443

Helfrich K 1972 Theor. Chim. Acta 24271

Herrick D E 1975 Phys. Rev. A 121949

1983 Adv. Chem. Phys. 521

Herrick D E and Kellman M E 1980 Phys. Rev. A 21418

Herrick D E and Sinanoglu O 1975 Phys. Rev A 1197

Hiskes J R, Thrter C B and Moody D A 1964 Phys. Rev. 133 A424

Ho Y K 1983 Phys. Rep. 991

— 1986 Phys. Rev. A 344402

Iwasaki M et al 1991 Phys. Rev. Lett. 671246

James H M and Coolidge A S 1937 Phys. Rev 51857

Jones R R and Gallagher T F 1990 Phys. Rev. A 422655

Junker B R 1982 Adv. At. Mol Phys. 18207

Komarov I V and Slavyanov S Y 1968 J. Phys. B: At. Mol. Phys. 11066

Landau L D and Lifshitz E M 1965 Quantum Mechanics: Non-Relativistic Theory 3rd edn (Oxford: Pergamon)

Lichtenberg A J and Lieberman M A 1983 Regular and Stochastic Motion (New York: Springer)

Lin C D 1984 Phys. Rev: A 291019

- 1986 Adv. At. Mol. Phys. 2077

Macek J 1968 J. Phys. B: At. Mol. Phys. 1831

Miller W H $1975 \mathrm{~J}$. Chem. P/hys. 63996

Molina Q 1989 Phys. Rev. A 393298

Pack R T and Hirschfelder J O 1968 J. Chem. Phys. 494009

Pekeris C L 1958 Phys. Rev. 1121649

Percival I C 1977 Proc. $R$ Soc. A 353289

Power J D 1973 Proc. R. Soc. A 274663

Purcell E M 1976 Berklcy Physics Course vol 2 (New York: McGraw-Hill) p 61

Rau A R P 1983 J. Phys. B: At. Mol Phys. 16 L699

Reinhardt W P 1982 Ann. Rev. Phys. Chem. 33223

Richter K 1991 PhD Thesis Universität Freiburg, unpublished

Richter R, Rost J M, Thürwächter R, Briggs J S, Wintgen D and Solov'ev E A 1991 Phys. Rev. Let. 66 149

Richter K and Wintgen D 1990a Phys. Rev. Lett. 651965

- 1990b J. Phys. B: At. Mol Opt. Phys. 23 L197

1991 J. Phys. Bi At. MoL Opt. Phys. 24 L565

Rost J M and Briggs J S 1989 J. Phys. B: At. Mol. Opt. Phys. 223587

1991 J. Phys. B: At. Mol Opt. Phys. 244293

Rost J M, Gersbacher R, Richter K, Briggs J S and Wintgen D 1991 J. Phys. B: At. Mol Opt Phys. 24 2455

Roussel F, Cheret M, Chen L, Bolzinger T, Spiess G, Hare J and Gross M 1990 Phys. Rev. Lett. 653112 
Sadeghpour H R and Greene C H 1990 Phys. Rev. Leth 65313

Satin A 1978 Comput. Phys. Commun. 14121

Slater J C 1977 Quantum Theory of Matter (Huntington: Krieger) ch 20

Smilansky U 1992 Proc. 1989 Les Houches Summer School on 'Quantum Chaos' eds M J Giannoni et al (Amsterdam: Elsevier) p 371

Solov'ev E A 1981 Sov. Phys.-JETP 54893

—_ 1985 Sov. Phys.-JETP 621148

Thürwächter R 1992 PhD Thesis Universität Freiburg, in preparation

Wintgen D, Marxer H, Richter K and Tanner $G$ 1992a in preparation

Wintgen D, Richter K and Tanner G 1992b CHAOS 219

Yamazaki T 1992 Z Phys. A at press

Yamazaki T et al 1989 Phys. Rev. Lett 631590

Zhen Z 1990 Phys. Rev A 4187 